\title{
Synthese und Reaktionen optisch aktiver Cyanhydrine
}

\author{
Franz Effenberger *
}

Bei der großen Bedeutung, die Cyanhydrine in der Technik und in der Organischen Chemie immer schon hatten, ist es erstaunlich, daß optisch aktive Cyanhydrine erst in den letzten Jahren intensiver untersucht und in der Synthese eingesetzt wurden. Der Grund dafür ist wohl darin zu sehen, daß es erst in neuerer Zeit gelungen ist, chirale Cyanhydrine, hauptsächlich mit enzymatischen Methoden, relativ einfach und in hoher optischer Reinheit herzustellen. Chirale Cyanhydrine sind, zumeist als Glycoside geschützt, in der Natur weit verbreitet. Annähernd dreitausend Pflanzen und vielen Insekten dienen sie unter anderem als Abwehrstoffe gegen "Fresser". Ihre große Bedeutung in der Organischen Chemie beruht auf ihrem enormen Syn- thesepotential für die Gewinnung anderer wichtiger chiraler Verbindungen. In Wirkstoffen sind chirale Cyanhydrinbausteine nur vereinzelt enthalten. Wegen der Notwendigkeit, bei neuen Wirksubstanzen mit Chiralitätszentren, sämtliche Stereoisomere herzustellen und sie bezüglich ihres Wirkungsspektrums sowie ihres Metabolismus zu untersuchen, ist die Entwicklung neuer und einfacher stereoselektiver Synthesen erforderlich. Chirale Cyanhydrine können hierbei wertvolle Ausgangsverbindungen sein. Im vorliegenden Artikel werden folgende Themen behandelt: die durch die Enzyme $(R)$ - oder ( $S$ )-Oxynitrilase katalysierte enantioselektive Addition von Blausäure an Aldehyde und Ketone zu $(R)$ bzw. ( $S$ )-Cyanhydrinen; die durch cycli- sche Dipeptide katalysierte enantioselektive Addition von Blausäure an Aldehyde; die durch Lipasen und Esterasen katalysierte enantioselektive Hydrolyse bzw. Veresterung racemischer Cyanhydrine und Cyanhydrinester; Folgereaktionen der Nitrilgruppe chiraler Cyanhydrine $\mathrm{zu}$ optisch aktiven $\alpha$-Hydroxycarbonsäuren, $\alpha$-Hydroxyaldehyden, $\alpha$ Hydroxyketonen und 2-Aminoalkoholen; Folgereaktionen der OH-Gruppe chiraler Cyanhydrine durch Sulfonylaktivierung und anschließende stereoselektive Substitution mit Nucleophilen unter Konfigurationsumkehr zu optisch aktiven $\alpha$-Azidonitrilen, $\alpha$-Aminonitrilen und $\alpha$-Fluornitrilen.

\section{Einleitung}

In den letzten Jahren wurde eine Reihe von Verfahren zur Herstellung optisch aktiver Cyanhydrine entwickelt, da über diese andere wichtige Klassen optisch aktiver Verbindungen, z.B. $\alpha-$ Hydroxycarbonsäuren, $\alpha$-Hydroxyaldehyde und 2-Aminoalkohole, leicht zu erhalten sind ${ }^{[1-3]}$.

Bei den bisher beschriebenen stereoselektiven Synthesen von Cyanhydrinen kann zwischen chemischen Methoden und enzymkatalysierten Verfahren unterschieden werden. Die wichtigsten chemischen Methoden sind die diastereoselektive Addition von Trimethylsilylcyanid ${ }^{[4]}$ oder von vergleichbaren anderen Cyanierungsagentien ${ }^{[5]}$ an optisch aktive Aldehyde und die enantioselektive Addition von Trimethylsilylcyanid an Aldehyde in Gegenwart chiraler Katalysatoren ${ }^{[6,7]}$. Enzymkatalysiert sind optisch aktive Cyanhydrine zum einen über die durch Oxynitrilasen katalysierte enantioselektive Addition von Blausäure an Aldehyde und zum anderen über enantioselektive Verseifungen racemischer Cyanhydrinester oder enantioselektive Veresterun-

[*] Prof. Dr. F. Effenberger

Institut für Organische Chemie der Universität

Pfaffenwaldring 55, D-70569 Stuttgart

Telefax: Int. $+711 / 685-4269$ gen racemischer Cyanhydrine mit Lipasen bzw. Esterasen zugänglich.

Bei dieser Unterteilung in chemische und enzymatische Synthesemethoden ist die Zuordnung des Verfahrens unter Verwendung cyclischer Dipeptide, die in speziellen Fällen eine enantioselektive Addition von Blausäure an Aldehyde katalysieren, nicht ohne weiteres möglich ${ }^{[8]}$. Formal sind cyclische Dipeptide zwar als chirale Katalysatoren anzusehen, eine strukturelle Ähnlichkeit mit den aktiven Zentren in Oxynitrilasen, deren Struktur noch völlig unbekannt ist, kann jedoch nicht ausgeschlossen werden.

Ein Vergleich der Vor- und Nachteile der chemischen mit denen der enzymatischen Methoden zur allgemeinen Synthese optisch aktiver Cyanhydrine fällt eindeutig zugunsten der enzymkatalysierten Verfahren aus und zwar sowohl bezüglich der erreichbaren optischen Ausbeuten, als auch bezüglich der Herstellung größerer Mengen an Cyanhydrinen. Dies schließt nicht aus, daß in einigen Fällen auch andere Verfahren vorteilhaft sein können.

In diesem Übersichtsartikel werden ausschließlich die enzymkatalysierten Verfahren und die Anwendung cyclischer Dipeptide als Katalysatoren zur Synthese optisch aktiver Cyanhydrine zusammengefaßt und diskutiert, wobei ihrer Bedeutung entsprechend Aldehyd-Cyanhydrine im Vordergrund stehen. Darüber hinaus wird das große Synthesepotential der optisch aktiven 
Cyanhydrine für die Synthese anderer wichtiger chiraler Verbindungen aufgezeigt.

\section{2. (R)-Oxynitrilase-katalysierte Additionen von Blausäure an Aldehyde}

Die von Rosenthaler 1908 publizierte Synthese von Mandelsäurenitril aus Benzaldehyd, Blausäure und Emulsin als Enzymquelle ist wahrscheinlich eine der ersten „Durch Enzyme bewirkten asymmetrischen Synthesen "[9]. Obwohl schon von Rosenthaler recht genaue Angaben über den Ablauf der Reaktion und den EinfluB der Reaktionsbedingungen auf die optische Ausbeute gemacht wurden, fand dieser äußerst interessante Ansatz einer enzymkatalysierten asymmetrischen Synthese nur wenig Beachtung ${ }^{[10]}$.

Erst mehr als fünfzig Jahre später wurden diese Untersuchungen von Pfeil et al. wieder aufgenommen und die Methode erfolgreich weiterentwickelt. Das im Emulsin vorhandene, die Addition von Blausäure an Benzaldehyd katalysierende Enzym (R)-Oxynitrilase [EC 4.1.2.10] wurde aus Bittermandeln (Prunus amygdalus) isoliert, gereinigt und charakterisiert ${ }^{[11]}$. Es wurde gezeigt, daß die $(R)$-Oxynitrilase neben dem natürlichen Substrat Benzaldehyd auch andere aromatische sowie aliphatische, ungesättigte und heterocyclische Aldehyde als Substrate akzeptiert und die Bildung der entsprechenden $(R)$-Cyanhydrine katalysiert $^{[12]}$. Die über die Drehwerte bestimmten optischen Ausbeuten waren beim $(R)$-Mandelsäurenitril mit $86 \%$ Enantiomerenüberschuß (ee) für damalige Verhältnisse hoch. Bei langsam reagierenden Aldehyden waren die optischen Ausbeuten deutlich niedriger ${ }^{[12]}$, so daß diese sehr einfache Synthese von (R)-Cyanhydrinen in den nachfolgenden zwei Jahrzehnten praktisch keine Anwendung und Beachtung fand.

Alle Bemühungen, die optischen Ausbeuten unter den von Pfeil et al. angewendeten Reaktionsbedingungen - Wasser oder Wasser/Ethanol als Lösungsmittel und ein $\mathrm{pH}$-Wert von etwa 5 , der dem Wirkungsoptimum des Enzyms entspricht ${ }^{[12 b]}$ - zu verbessern, waren erfolglos ${ }^{[13 a]}$. Unter diesen Bedingungen ließ sich die chemische Addition von Blausäure an Aldehyde, die zu racemischen Produkten und damit zu einer Verschlechterung der optischen Ausbeute bei der enzymatischen Synthese führt, nicht unterdrücken. Erwartungsgemäß macht sich dieser Nachteil besonders bei den Aldehyden bemerkbar, die enzymkatalysiert langsam reagieren.
Ein entscheidender Durchbruch gelang, als gezeigt werden konnte, daß die unerwünschte chemische Addition der Blausäure an Aldehyde weitgehend unterdrückt werden kann, wenn die Reaktionen in mit Wasser nicht mischbaren organischen Lösungsmitteln, z.B. Ethylacetat oder Diisopropylether, durchgeführt werden (Abb. 1) ${ }^{[13]}$. Da, wie Abbildung 1 zeigt, die unka-

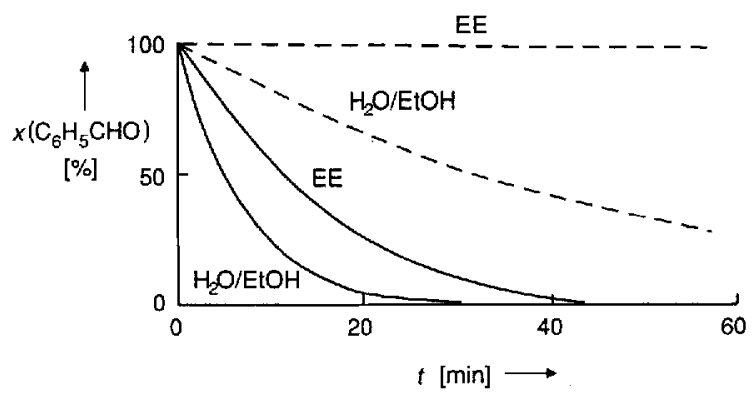

Abb. 1. Vergleich der Reaktionsgeschwindigkeiten der chemischen (-....) und enzymatischen (-) Additionen von $\mathrm{HCN}$ an Benzaldehyd ( $x=$ Molenbruch [\%]) in $\mathrm{H}_{2} \mathrm{O} / \mathrm{EtOH}$ und in Ethylacetat (EE).

talysierte Addition von Blausäure an Benzaldehyd in Ethylacetat nicht oder nur sehr langsam erfolgt, werden bei Aldehyden, die schlechte Substrate für das Enzym sind und die deshalb auch enzymkatalysiert langsam reagieren, trotzdem hohe optische Ausbeuten erreicht (Schema 1, Tabelle 1) ${ }^{[13]} .(R)-$

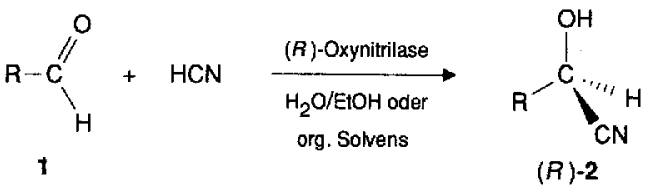

Schema 1. Synthese von (R)-Cyanhydrinen durch $(R)$-Oxynitrilase-katalysierte Addition von $\mathrm{HCN}$ an Aldehyde.

Oxynitrilase zeigt die bei Enzymen nicht so häufig anzutreffende vorteilhafte Eigenschaft, daß sie trotz geringer Substratselektivität Produkte mit hoher optischer Reinheit liefert. So werden von $\operatorname{der}(R)$-Oxynitrilase aromatische und aliphatische Aldehyde gleichermaßen akzeptiert und enantioselektiv mit HCN um-

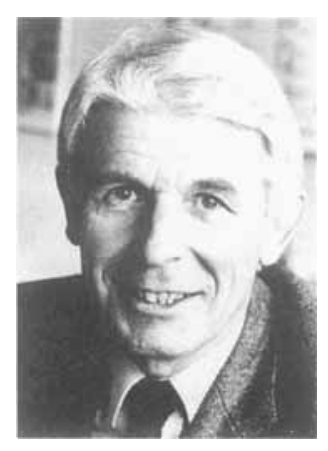

Frunz Effenberger, geboren 1930 in Goldenstein (Nordmähren), studierte von 1951 bis 1956 Chemie an der Technischen Hochschule Stuttgart und promovierte 1959 in Stuttgart bei H. Bredereck. 1964 habilitierte er sich in Stuttgart und ging dann für ein Jahr zu P. A. S. Smith an die University of Michigan, Ann Arbor. 1972 wurde er als Nachfolger von H. Bredereck an die Universität Stuttgart berufen. Er war von 1964 bis 1969 Winnacker-Stipendiat. 1977 nahm er eine Gastprofessur an der Cornell University in Ithaca und 1989 eine an der Ecole Superieure de Physique et Chimie Industrielles in Paris wahr. 1991 wurde er mit dem Alexander-von-Humboldt-Forschungspreis ausgezeichnet. Seine Arbeitsgebiete sind präparative und mechanistische Untersuchungen an elektronenreichen $\pi$-Systemen (Enolethern, Aminobenzolen, Donor-Acceptor-substituierten Polyenen und Oligothiophenen), Synthesen und Reaktionen von $\alpha$-Aminosäuren, die Anwendung von Enzymreaktionen in der organischen Synthese mit Schwerpunkt stereoselektive C-C-Verknüpfungsreaktionen. 
Tabelle 1. Synthese der $(R)$-Cyanhydrine $(R)$-2 durch enzymkatalysierte Addition von $\mathrm{HCN}$ an Aldehyde 1 in $\mathrm{H}_{2} \mathrm{O} / \mathrm{EtOH}$ und in organischen Lösungsmitteln [13].

\begin{tabular}{|c|c|c|c|c|c|c|c|c|c|}
\hline \multirow[t]{2}{*}{$\mathrm{R}$} & \multicolumn{3}{|c|}{ in $\mathrm{H}_{2} \mathrm{O} / \mathrm{EtOH}$} & \multicolumn{3}{|c|}{ in Ethylacetat/Avicel [a] } & \multicolumn{3}{|c|}{$\begin{array}{l}\text { in Diisopropylether } \\
\text { Avicel [a] }\end{array}$} \\
\hline & $\begin{array}{l}t \\
{[\mathrm{~b}]}\end{array}$ & $\begin{array}{l}\text { Ausb. } \\
{[\%]}\end{array}$ & $\begin{array}{l}e e[b] \\
{[\%]}\end{array}$ & $\begin{array}{l}t \\
{[\mathrm{~h}]}\end{array}$ & $\begin{array}{l}\text { Ausb. } \\
{[\%]}\end{array}$ & $\begin{array}{l}\text { ee }[\mathrm{b}] \\
{[\%]}\end{array}$ & $\begin{array}{l}l \\
{[\mathrm{~h}]}\end{array}$ & $\begin{array}{l}\text { Ausb. } \\
{[\%]}\end{array}$ & $\begin{array}{l}\text { ee }[\mathrm{b}] \\
{[\%]}\end{array}$ \\
\hline $\mathrm{C}_{6} \mathrm{H}_{5}$ & 1 & 99 & 86 & 2.5 & 95 & 99 & 3 & 96 & $>99$ \\
\hline $3-\mathrm{C}_{6} \mathrm{H}_{5} \mathrm{O}-\mathrm{C}_{6} \mathrm{H}_{4}$ & 5 & 99 & 11 & 192 & 99 & 98 & - & .. & - \\
\hline 2-Furyl & 2 & 86 & 69 & 4 & 88 & 99 & & & \\
\hline 3-Thienyl & - & - & & & - & - & 6 & 95 & $>99$ \\
\hline 3-Pyridyl & 2.5 & 78 & 7 & 4.5 & 89 & 14 & 3 & 97 & 82 \\
\hline $\mathrm{H}_{3} \mathrm{CCH}=\mathrm{CH}$ & 1.5 & 68 & 76 & 3 & 68 & 97 & - & - & - \\
\hline $\mathrm{H}_{3} \mathrm{CS}\left(\mathrm{CH}_{2}\right)_{2}$ & 3 & 87 & 60 & 6.5 & 97 & 80 & 16 & 98 & 96 \\
\hline $\mathrm{C}_{3} \mathrm{H}_{7}$ & 2 & 75 & 69 & - & - & - & 16 & 98.6 & 98 \\
\hline $\mathrm{C}_{6} \mathrm{H}_{5}\left(\mathrm{CH}_{2}\right)_{3}$ & - & - & - & - & - & - & 45 & 94 & 90 \\
\hline$\left(\mathrm{H}_{3} \mathrm{C}\right)_{3} \mathrm{C}$ & 2.5 & 56 & 45 & 4.5 & 78 & 73 & 4.5 & 84 & 83 \\
\hline
\end{tabular}

[a] Das Enzym wurde auf einer kristallinen Cellulose (Avicel) gebunden eingesetzt. [b] Nach Umselzung mit (R)- $\alpha$-Methoxy- $\alpha$-trifluormethylphenylessigsäurechlorid $[(R)$ $(+)$-MTPA-Chlorid] zu den diastereomeren $(R)-(+)$-MTPA-Estern oder nach Acetylierung mit Acetanhydrid gaschromatographisch bestimmt [13].

gesetzt (Tabelle 1). Selbst bei sterisch anspruchsvollen Edukten wie Isopentylaldehyd werden hohe optische Ausbeuten ( $83 \%$ ee) erreicht. Am Beispiel der Umsetzung von 3-Phenoxybenzaldehyd wird der Vorteil des organischen Lösungsmittels gegenüber dem wäßrigen System besonders deutlich: Selbst bei einer Reaktionszeit von 192 Stunden ist die unkatalysierte Addition so stark unterdrückt, daß das entsprechende $(R)$-Cyanhydrin mit $98 \%$ ee erhalten wurde (Tabelle 1). Eine Ausnahme bei diesen Reaktionen ist Pyridin-3-carbaldehyd, der in Ethylacetat als Lösungsmittel nur niedrige optische Ausbeuten $(14 \%$ ee $)$ ergab. Die Produktbildung kann auch von der Art des organischen Lösungsmittels abhängen, was am Beispiel der Reaktion von Pyridin-3-carbaldehyd besonders deutlich wird (Tabelle 1).

Über die Verwendung organischer Lösungsmittel bei enzymkatalysierten Reaktionen gibt es viele Publikationen ${ }^{[14]}$. Besonders intensiv wurden Reaktionen untersucht, in denen Wasser als Reaktionspartner auftritt. Dazu gehören insbesondere die durch Lipasen, Amidasen und Proteasen katalysierten Ester-, Amid- bzw. Peptidbindungsknüpfungen und -spaltungen ${ }^{[14]}$. Bei der durch (R)-Oxynitrilase katalysierten Cyanhydrinbildung ist Wasser kein Reaktionspartner; es begünstigt jedoch offensichtlich die nichtenzymatische Addition von Blausäure an die Carbonylgruppe.

Unter den vielen organischen Lösungsmitteln, die im Zusammenhang mit den Oxynitrilase-katalysierten Reaktionen untersucht wurden ${ }^{[15]}$, ist Diisopropylether bezüglich der erreichbaren optischen Ausbeuten besonders günstig, wie beispielhaft aus einem Vergleich einiger der in Tabelle 1 angeführten Reaktionen in Ethylacetat mit denen in Diisopropylether hervorgeht. Darüber hinaus bleibt in Diisopropylether als Lösungsmittel die Enzymaktivität über mehrere Wochen weitgehend erhalten (Abb. 2), was von großer Bedeutung für die praktische Anwendung eines Enzyms ist. In Ethylacetat als Lösungsmittel wird dagegen schon nach wenigen Stunden ein Aktivitätsabfall der (R)-Oxynitrilase um mehr als die Hälfte festgestellt (Abb. 2). Der im Vergleich mit Ethylacetat geringere Wassergehalt des Diisopropylethers ${ }^{[16]}$ wirkt sich offensichtlich günstig auf die Aktivität und die Stabilität der $(R)$-Oxynitrilase aus.

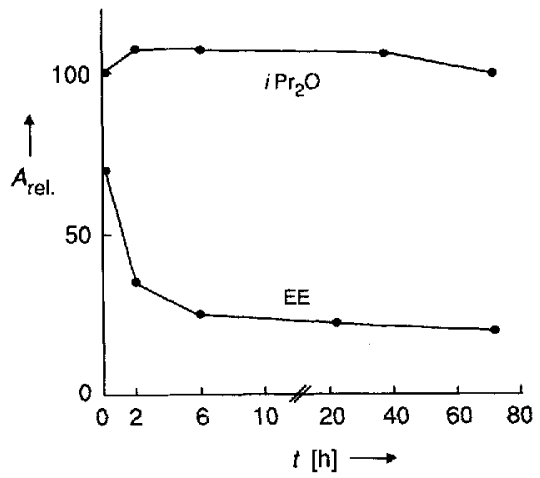

Abb. 2. Zeitabhängigkeit der relativen Aktivität $A$ von $(R)$-Oxynitrilase auf einer kristallinen Cellulose (Avicel) in Ethylacetat (EE) und in Diisopropylether ( $\mathrm{Pr}_{2} \mathrm{O}$ ) [15a].

Für die praktische Durchführung der Reaktionen in organischen Lösungsmitteln ist es besonders vorteilhaft, daß das Enzym auf einem geeigneten Trägermaterial, z. B. einer kristallinen Cellulose wie Avicel, adsorptiv so fest gebunden wird, daß nach beendeter Reaktion vom ,trägerfixierten Enzym" abfiltriert und der Katalysator erneut eingesetzt werden kann. Ein weiterer Vorteil der Reaktionen mit $(R)$-Oxynitrilasen in organischen Lösungsmitteln ist darin zu sehen, daß mit relativ hohen Substratkonzentrationen (bis $2 \mathrm{molL}^{-1}$ ) ohne merkliche Minderung der optischen Ausbeuten gearbeitet werden kann, woraus sich außerordentlich günstige Raum-Zeit-Ausbeuten für die Produktbildung ergeben ${ }^{[16]}$. Die Reaktionen können auch kontinuierlich durchgeführt werden, indem man die Edukte entweder im organischen Solvens gelöst über eine Säule schickt, die mit dem trägerfixierten Enzym beladen ist ${ }^{[15 a]}$, oder in einem Membranreaktor reagieren läßt, wobei die Produkte von den Edukten und dem Katalysator durch Evaporation abgetrennt werden $^{[15 b]}$.

Durch Erniedrigung des pH-Wertes unter $\mathrm{pH} 4.0$ konnten Kula et al. ${ }^{[17]}$ bei der durch Oxynitrilasen katalysierten Addition von Blausäure an Aldehyde auch im wäßrigen Medium hohe optische Ausbeuten erreichen. Im sauren Medium wird ebenfalls die chemische Addition von Blausäure an Aldehyde unterbunden, so daß sich bessere optische Ausbeuten ergeben. Bei dieser Arbeitsweise ist die Enzymaktivität wegen des niedrigeren $\mathrm{pH}$-Werts deutlich verringert, was durch Verwendung größerer Enzymmengen zwar kompensiert werden kann, bei langsam reagierenden Aldehyden jedoch trotzdem zu schlechten optischen Ausbeuten führt. Aldehyde, die in Wasser schwer oder nicht löslich sind, lassen sich auf diese Weise nicht enzymkatalysiert umsetzen.

Wegen der Toxizität der Blausäure fehlte und fehlt es nicht an Versuchen, freie Blausäure bei der Synthese der Cyanhydrine zu vermeiden. So wurden Aldehyde in Wasser/Ethanol in Gegenwart von $(R)$-Oxynitrilase direkt mit $\mathrm{KCN}$ und Essigsäure zu (R)-Cyanhydrinen umgesetzt, wobei allerdings schlechtere optische Ausbeuten erzielt wurden ${ }^{[18]}$. Transcyanierungen von Aldehyden mit Aceton-Cyanhydrin als HCN-Donor in Gegenwart von $(R)$-Oxynitrilase unter Bildung von $(R)$-Aldehyd-Cyanhydrinen und Aceton sind beschrieben ${ }^{[19]}$, die Ergebnisse konnten jedoch bezüglich der optischen Ausbeuten nicht nachvollzogen werden ${ }^{[20]}$. 


\section{3. (S)-Oxynitrilase-katalysierte Addition von Blausäure an Aldehyde}

Eine Oxynitrilase, die bevorzugt $(S)$-Cyanhydrine in Aldehyde und Blausäure spaltet, und die damit auch die Umkehrreaktion katalysieren müßte, wurde erstmals 1961 von Conn und Bové aus Sorghum bicolor isoliert und charakterisiert ${ }^{[21 a]}$. Diese (S)-Oxynitrilase [EC 4.1.2.11] und die aus Bittermandeln isolierten $(R)$-Oxynitrilase [EC 4.1.2.10] unterscheiden sich signifikant sowohl in ihrer Struktur ${ }^{[11 b, 21 b, 22]}$ als auch in ihren Katalysatoreigenschaften. Der auffälligste Strukturunterschied besteht darin, daß die $(R)$-Oxynitrilase eine prosthetische Gruppe [Flavinadenindinucleotid (FAD)] hat, die bei der $(S)$ Oxynitrilase fehlt. Durch Abspalten des FAD verliert die $(R)$ Oxynitrilase vollständig ihre katalytische Aktivität.

Die Gewinnung größerer, für präparative Anwendungen ausreichender Mengen an (S)-Oxynitrilase aus Sorghum bicolor ist wesentlich aufwendiger als die Gewinnung von $(R)$-Oxynitrilase aus Bittermandeln. Vermutlich war dies auch der entscheidende Grund dafür, daß dieses Enzym erst in jüngster Zeit in der organischen Synthese verwendet wurde ${ }^{[23,24]}$.

(S)-Oxynitrilase aus Sorghum katalysiert ausschließlich die Addition von Blausäure an aromatische und heterocyclische Aldehyde unter Bildung der entsprechenden $(S)$-Cyanhydrine; aliphatische Aldehyde sind keine Substrate für dieses Enzym $^{[23,24]}$. Umsetzungen mit der (S)-Oxynitrilase als Katalysator ergeben in organischen Lösungsmitteln ähnliche optische Ausbeuten (Schema 2, Tabelle 2) ${ }^{[23]}$ wie die vergleichbaren Reaktionen mit der ( $R$ )-Oxynitrilase (Tabelle 1).
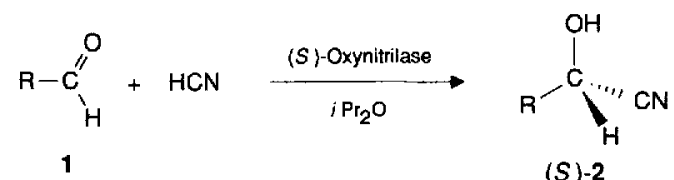

(S)-2

Schema 2. Synthese von (S)-Cyanhydrinen.

Tabelle 2. Synthese der (S)-Cyanhydrine $(S)$-2 durch enzymkatalysierte Addition von HCN an Aldehyde 1 in Diisopropylether [23].

\begin{tabular}{|c|c|c|c|c|c|c|c|}
\hline \multirow[t]{2}{*}{$\mathrm{R}$} & \multicolumn{3}{|c|}{ (S)-Cyanhydrin 2} & \multirow[t]{2}{*}{$\mathrm{R}$} & \multicolumn{3}{|c|}{$(S)$-Cyanhydrin 2} \\
\hline & $\begin{array}{l}i \\
{[\mathrm{~h}]}\end{array}$ & $\begin{array}{l}\text { Ausb } \\
{[\%]}\end{array}$ & $\begin{array}{l}\mathcal{e e}[\mathrm{a}] \\
{[\%]}\end{array}$ & & $\begin{array}{l}t \\
{[\mathrm{~h}]}\end{array}$ & $\begin{array}{l}\text { Ausb. } \\
{[\%]}\end{array}$ & $\begin{array}{l}e c[a] \\
{[\%]}\end{array}$ \\
\hline $\mathrm{C}_{6} \mathrm{H}_{5}$ & 3 & 91 & 97 & $3-\mathrm{F}_{3} \mathrm{CC}_{6} \mathrm{H}_{4}$ & 20 & 87 & 52 \\
\hline $4-\mathrm{ClC}_{6} \mathrm{H}_{4}$ & 48 & 87 & 54 & $3-\mathrm{H}_{3} \mathrm{COC}_{6} \mathrm{H}_{4}$ & 20 & 93 & 89 \\
\hline $4-\mathrm{H}_{3} \mathrm{CC}_{6} \mathrm{H}_{4}$ & 32 & 78 & 87 & $3-\mathrm{C}_{6} \mathrm{H}_{5} \mathrm{OC}_{6} \mathrm{H}_{4}$ & 144 & 93 & 96 \\
\hline $3-\mathrm{HOC}_{6} \mathrm{H}_{4}$ & 24 & 97 & 91 & 2-Furyl & 9 & 80 & 80 \\
\hline $3-\mathrm{BrC}_{6} \mathrm{H}_{4}$ & 18 & 94 & 92 & 3-Thienyl & 20 & 85 & 97 \\
\hline $3-\mathrm{ClC}_{6} \mathrm{H}_{4}$ & 48 & 95 & 91 & & & & \\
\hline
\end{tabular}

[a] Analog Tabelle 1 gaschromatographisch als $(R)$-MTPA-Ester bestimmt.

Bei der Synthese der $(S)$-Cyanhydrine ist das Arbeiten in organischen Lösungsmitteln gegenüber dem Arbeiten im wäBrigen Medium aus zwei Gründen besonders vorteilhaft: Zum einen sind in organischen Lösungsmitteln wesentlich kleinere Mengen des schlechter zugänglichen Enzyms erforderlich und zum anderen lassen sich einige technisch interessante $(S)$-Cyanhydrine (z.B. 3-Phenoxybenzaldehyd sowic heterocyclische Aldehyde) wegen der Schwerlöslichkeit der Ausgangsaldehyde im wäßrigen System nur schwierig herstellen ${ }^{[24]}$.

Kürzlich wurde über die katalytische Wirkung einer weiteren $(S)$-Oxynitrilase berichtet ${ }^{[25]}$, die aus den Blättern von Hevea brasiliensis (Gummibaum) isoliert wurde. Anders als die $(S)$ Oxynitrilase aus Sorghum akzeptiert dieses Enzym neben aromatischen auch aliphatische Aldehyde als Substrate, wobei die optischen Ausbeuten bei einigen Substraten allerdings unbefriedigend sind ${ }^{[25]}$.

Trotz der Verbesserungen und Optimierungen, die bei der Gewinnung und Isolierung von $(S)$-Oxynitrilase aus Sorghum erreicht wurden ${ }^{[26]}$, wird für technische Anwendungen eine Klonierung und Überexpression des Gens der (S)-Oxynitrilase in pro- oder eukaryotischen Produktionsorganismen erforderlich sein. In den bisherigen Untersuchungen zur Lösung dieses Problems, das für Anwendungen dieses Enzyms ausschlaggebend sein wird, konnte die Klonierung der $(S)$-Oxynitrilase aus Sorghum bicolor L. schon weitgehend ( $90 \%$ der nativen Hydroxynitril-Lyase (HNL)) verwirklicht werden ${ }^{[26 b]}$.

\section{4. (R)-Oxynitrilase-katalysierte Addition von Blausäure an Ketone}

Nur wenige optisch aktive Keton-Cyanhydrine sind beschrieben. In den meisten Fällen wurden sie durch stereoselektive Addition von Blausäure oder von Cyaniden an Verbindungen mit einem Chiralitätszentrum erhalten. So ist die Addition von $\mathrm{HCN}$ an 17-Oxosteroide hoch diastereoselektiv ${ }^{[27]}$. Ebenfalls hohe optische Induktionen werden bei den Additionen von $\mathrm{Me}_{2} \mathrm{Si}(\mathrm{CN})_{2}$ an chirale $\beta$-Hydroxyketone ${ }^{[28]}$, von $\mathrm{Et}_{2} \mathrm{AlCN}$ an chirale $\beta$-Ketosulfoxide ${ }^{[29]}$ sowie von Trimethyl-(2-propenyl)silan an optisch aktive Acylcyanide ${ }^{[30]}$ erhalten. Weniger erfolgreich bezüglich der chemischen Ausbeuten verläuft die enantioselektive Hydrolyse racemischer Ester von Keton-Cyanhydrinen mit Hefezellen von Pichia miso ${ }^{[31]}$.

Der als Cyanogenese bezeichnete Abwehrmechanismus vieler Pflanzen und einiger Insekten, bei dem enzymkatalysiert Blausäure aus glykosidgeschützten Cyanhydrinen freigesetzt wird, ist nicht auf die Spaltung von Aldehyd-Cyanhydrinen beschränkt, sondern wird auch für Keton-Cyanhydrine beschrieben ${ }^{[32]}$. Es war deshalb zu erwarten, daß auch Ketone Substrate für Oxynitrilasen sein können. ( $R$ )-Oxynitrilase aus Bittermandeln [EC 4.1.2.10], für die Benzaldehyd das natürliche Substrat ist, katalysiert überraschenderweise auch die enantioselektive Addition von Blausäure an Ketone unter Bildung der $(R)$ Keton-Cyanhydrine ${ }^{[33]}$ (Schema 3). 2-Alkanone reagieren dabei in guten chemischen und sehr guten optischen Ausbeu-

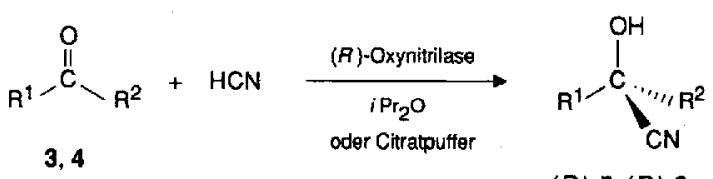

$(R)-5,(R)-6$ 
ten ${ }^{[33 i]} ; 3$-Alkanone sind deutlich schlechtere Substrate, die unbefriedigende chemische Ausbeuten bei gleichzeitig verminderter optischer Reinheit ergeben (Tabelle 3) ${ }^{[33]}$. Bei der Synthese

Tabelle 3. Synthese der $(R)$-Keton-Cyanhydrine $(R)-5\left(\mathrm{R}^{2}=\mathrm{CH}_{3}\right)$ und $(R)-6$ $\left(\mathrm{R}^{2}=\mathrm{C}_{2} \mathrm{H}_{5}\right)$ durch enzymkatalysierte Addition von $\mathrm{HCN}$ an die Ketone 3 $\left(\mathrm{R}^{2}=\mathrm{CH}_{3}\right)$ bzw. $\mathbf{4}\left(\mathrm{R}^{2}=\mathrm{C}_{2} \mathrm{H}_{5}\right)$ in Diisopropylether [33a] und in wäßrigem Citratpuffer [33 b].

\begin{tabular}{|c|c|c|c|c|c|c|}
\hline \multirow[t]{2}{*}{$\mathrm{R}^{\prime}$} & \multicolumn{4}{|c|}{$\begin{array}{l}\text { in Diisopropylether/Avicel [a] } \\
\begin{array}{lr}(R)-5[\mathrm{c}] & (R)-6[\mathrm{~d}]\end{array}\end{array}$} & \multicolumn{2}{|c|}{$\begin{array}{l}\text { in Citratpuffer }[\mathrm{b}] \\
(R)-5[\mathrm{c}]\end{array}$} \\
\hline & $\begin{array}{l}\text { Ausb. } \\
{[\%]}\end{array}$ & {$[\%]$} & $\begin{array}{l}\text { Ausb. } \\
{[\%]}\end{array}$ & $\begin{array}{l}e e \\
{[\%]}\end{array}$ & $\begin{array}{l}\text { Ausb. } \\
{[\%]}\end{array}$ & {$[\%]$} \\
\hline$n \mathrm{C}_{3} \mathrm{H}_{7}$ & 70 & 97 & 33 & 85 & 78 & 95 \\
\hline$n \mathrm{C}_{4} \mathrm{H}_{9}$ & 90 & 98 & 21 & 90 & 94 & 98 \\
\hline$n \mathrm{C}_{5} \mathrm{H}_{11}$ & 88 & 98 & 7 & 66 & 56 & 96 \\
\hline$\left(\mathrm{H}_{3} \mathrm{C}\right)_{2} \mathrm{CHCH}_{2}$ & 57 & 98 & - & - & 40 & 98 \\
\hline
\end{tabular}

[a] $20 \mathrm{U}$ Enzym pro mmol Keton. [b] $50 \mathrm{U}$ Enzym pro mmol Keton. [c] Enantiomerenüberschüsse der diastereomeren $(R)$ - oder (S)-MTPA-Ester gaschromatogräphisch bestimmt [33]. [d] Enantiomerenüberschüsse nach Verseifung zu den entsprechenden Carbonsäuren und Veresterung mit Diazomethan gaschromatographisch auf $\beta$-Cyclodextrin-Säulen bestimmt [33b].

von $(R)$-Keton-Cyanhydrinen werden die besten Ergebnisse zwar ebenfalls beim Arbeiten in organischen Lösungsmitteln erreicht, die optischen Ausbeuten bei Umsetzungen in Citratpuffer $(\mathrm{pH} \leq 4)$ sind jedoch ähnlich groß, wenn mindestens die doppelte Menge Enzym eingesetzt wird (Tabelle 3) ${ }^{[33 \mathrm{~b}]}$.

Für die $(S)$-Oxynitrilase aus Sorghum bicolor sind Ketone nach den bisherigen Untersuchungen keine Substrate ${ }^{[24]}$.

\section{Cyclodipeptide als Katalysatoren für die enantioselektive Addition von Blausäure an Aldehyde}

Inoue et al, konnten zeigen, daß cyclische Dipeptide (1,4-Dioxopiperazine), die einen Histidinrest enthalten, die asymmetrische Addition von Blausäue an Benzaldehyd unter Bildung des optisch aktiven Mandelsäurenitrils katalysieren ${ }^{[8 a, b, 34]}$. Die höchste optische Ausbeute $(97 \%$ ee) wurde dabei mit dem Cyclodipeptid aus $(S)$-Histidin und $(S)$-Phenylalanin erzielt, wobei $(R)$-Mandelsäurenitril gebildet wird (Tabelle 4$)^{[34 c]}$. Die Reak-

Tabelle 4. Cyclische Dipeptide als Katalysatoren für die enantioselektive Addition von HCN an Benzaldehyd.

\begin{tabular}{|c|c|c|c|c|}
\hline Katalysator & Ausb. $[\%]$ & $\begin{array}{l}\text { Mandelsäurenitril } \\
\text { Konfiguration }\end{array}$ & $e e[\%]$ & Lit. \\
\hline $\operatorname{cyclo}[(S)-\mathrm{Ala}-(S)-\mathrm{His}]$ & 50 & $R$ & 9.9 & {$[8 \mathrm{a}, 34 \mathrm{a}]$} \\
\hline $\operatorname{cycho}((R)-\mathrm{Ala}-(S)-\mathrm{His}]$ & 90 & $R$ & 7.5 & {$[8 \mathrm{a}, 34 \mathrm{a}]$} \\
\hline $\operatorname{cyclo}[(S)-\mathrm{His}-(S)-\mathrm{His}]$ & 50 & $R$ & 2.5 & {$[8 \mathrm{a}, 34 \mathrm{a}]$} \\
\hline $\operatorname{cyclo}[(S)-\mathrm{His}-(S)$-Leu $]$ & 85 & $s$ & 55 & {$[34 \mathrm{~b}]$} \\
\hline cyclo[(S)-His-(S)-Phe] & 40 & $R$ & 90 & {$[8 \mathrm{~b}]$} \\
\hline cyclo $[(S)$-His- $(S)$-Phe $]$ & 97 & $R$ & 97 & {$[34 \mathrm{c}]$} \\
\hline $\operatorname{cyclo}[(R)$-His- $(R)$-Phe $]$ & 86 & $s$ & 79 & [36a] \\
\hline
\end{tabular}

tion ist nicht auf Benzaldehyd als Substrat beschränkt: Sie wurde auch auf andere aromatische Aldehyde und viele heterocyclische, sowie aliphatische Aldehyde angewendet, wobei jedoch häufig nur unbefriedigende optische Ausbeuten erzielt wurden (Tabel-
Tabelle 5. cyctol( $(S)$-His-( $S)$-Phe]-katalysierte enantioselektive Addition von $\mathrm{HCN}$ an Aldehyde 1 unter Bildung von $(R)$-Cyanhydrinen $(R)$-2.

\begin{tabular}{|c|c|c|c|c|c|c|c|}
\hline \multirow[t]{2}{*}{$\mathrm{R}$} & \multicolumn{2}{|c|}{$(R)-2$} & \multirow[t]{2}{*}{ Lit. } & \multirow[t]{2}{*}{$\mathrm{R}$} & \multicolumn{2}{|c|}{$(R)-2$} & \multirow[t]{2}{*}{ Lit. } \\
\hline & $\begin{array}{l}\text { Ausb. } \\
{[\%]}\end{array}$ & $\begin{array}{l}e r \\
{[\%]}\end{array}$ & & & $\begin{array}{l}\text { Ausb. } \\
{[\%]}\end{array}$ & $\begin{array}{l}e e \\
{[\%]}\end{array}$ & \\
\hline $3-\mathrm{C}_{6} \mathrm{H}_{5} \mathrm{OC}_{6} \mathrm{H}_{4}$ & 97 & 92 & {$[34 \mathrm{c}]$} & $4-\mathrm{F}_{3} \mathrm{CC}_{6} \mathrm{H}_{4}$ & 90 & 80 & [36b] \\
\hline $2-\mathrm{H}_{3} \mathrm{COC}_{6} \mathrm{H}_{4}$ & 45 & 84 & {$[34 \mathrm{c}]$} & $4-\left(\mathrm{CH}_{3}\right)_{2} \mathrm{NC}_{6} \mathrm{H}_{4}$ & 50 & 53 & [36b] \\
\hline $3-\mathrm{H}_{3} \mathrm{COC}_{6} \mathrm{H}_{4}$ & 97 & 90 & [36b] & 2-Naphthyl & 100 & 86 & [36b] \\
\hline $4-\mathrm{H}_{3} \mathrm{COC}_{6} \mathrm{H}_{4}$ & 85 & 83 & {$[36 \mathrm{~b}]$} & 2-Furyl & 76 & 93 & [36 b] \\
\hline 4- $\mathrm{H}_{3} \mathrm{CCC}_{6} \mathrm{H}_{4}$ & 91 & 92 & {$[36 \mathrm{~b}]$} & 2-Pyrrolyl & 31 & 0 & [36b] \\
\hline $4-\mathrm{O}_{2} \mathrm{NC}_{6} \mathrm{H}_{4}$ & 77 & 29 & {$[36 \mathrm{~b}]$} & 2-Thienyl & 70 & 58 & [36b] \\
\hline $3-\mathrm{HOC}_{6} \mathrm{H}_{4}$ & 75 & 67 & {$[35 \mathrm{~b}]$} & 3-Pyridyl & 89 & 0 & [36b] \\
\hline 4- $\mathrm{HOC}_{6} \mathrm{H}_{4}$ & 86 & 35 & {$[36 \mathrm{~b}]$} & $\mathrm{C}_{3} \mathrm{H}_{7}$ & 100 & 26 & [36b] \\
\hline 4- $\mathrm{ClC}_{6} \mathrm{H}_{4}$ & 96 & 66 & {$[36 \mathrm{~b}]$} & $\mathrm{C}_{6} \mathrm{H}_{5} \mathrm{CH}_{2}$ & 100 & 14 & {$[36 \mathrm{~b}]$} \\
\hline 4- $\mathrm{NCC}_{6} \mathrm{H}_{4}$ & 100 & 32 & {$[34 \mathrm{c}]$} & $\left(\mathrm{H}_{3} \mathrm{C}\right)_{2} \mathrm{CH}$ & 79 & 71 & [34c] \\
\hline 4- $\mathrm{C}_{6} \mathrm{H}_{5} \mathrm{OC}_{6} \mathrm{H}_{4}$ & 100 & 81 & [36 b] & $c-\mathrm{C}_{6} \mathrm{H}_{11}$ & 96 & 58 & [34c] \\
\hline
\end{tabular}

le 5$)^{[34 c, 35,36]}$. Ketone ergeben die entsprechenden Cyanhydrine mit nur sehr niedrigen optischen Ausbeuten ${ }^{[36 b]}$.

Cyclodipeptide mit $(S, S)$-Konfiguration katalysieren in nahezu allen untersuchten Fällen die Bildung von $(R)$-Cyanhydrinen im Überschuß. Einzige Ausnahme ist cyclo[(S)-His-(S)-Leu] als Katalysator, das bevorzugt (S)-Cyanhydrine bildet ${ }^{[34 \mathrm{~b}]}$. Wird statt cyclo[ $(S)$-His-( $S)$-Phe $]$ das Enantiomer cyclo[ $(R)$-His- $(R)$-Phe $]$ als Katalysator eingesetzt, so werden die $(S)$-Cyanhydrine im Überschuß gebildet (Tabelle 4) ${ }^{[36 a]}$. Diese Möglichkeit der Konfigurationsumkehr durch Variation des Katalysators wurde insbesondere zur Herstellung von $(S)$-3-Phenoxybenzaldehyd-Cyanhydrin genutzt ${ }^{[36,37]}$; dieses Cyanhydrin ist die Alkoholkomponente eines kommerziell bedeutenden Pyrethroids ${ }^{[38]}$.

Trotz umfangreicher Untersuchungen zum Mechanismus der durch cyclische Dipeptide katalysierten enantioselektiven Addition von Blausäure an Aldehyde sind noch keine gesicherten Aussagen über die Struktur des Übergangszustandes oder über Zwischenstufen und deren Struktur möglich ${ }^{[36 a}$, 39].

Auch acyclische Dipeptide katalysieren in beschränktem Maße die enantioselektive Addition von $\mathrm{HCN}$ an Aldehyde, wobei akzeptable optische Ausbeuten allerdings erst in Gegenwart spezieller Titan-Komplexe erzielt wurden ${ }^{[40]}$.

\section{Synthese von $(R)$ - und $(S)$-Cyanhydrinen durch kinetische Racematspaltung mit Esterasen und Lipasen}

Aldehyd-Cyanhydrine sind sekundäre Alkohole und bilden mit Carbonsäuren Ester. Damit eröffnet sich die prinzipielle Möglichkeit, racemische Gemische von Cyanhydrinen oder Cyanhydrinestern durch Lipase- oder Esterase-katalysierte Esterhydrolysen bzw. Veresterungen zu trennen ${ }^{[41]}$.

\subsection{Enantioselektive Hydrolyse racemischer Cyanhydrinester}

Bei der durch Esterhydrolasen aus $P$ seudomonas sp. katalysierten Verseifung racemischer Cyanhydrinacetate in Phosphatpuffer bei pH 7 können nur die nicht umgesetzten Cyanhydrinacetate in optisch aktiver Form isoliert werden; die durch Hydrolyse gebildeten chiralen Cyanhydrine racemisieren in der Regel unter diesen Bedingungen ${ }^{[42]}$. Nur in einigen Fällen lassen sich so auch die freien Cyanhydrine in optisch aktiver Form isolieren ${ }^{[43]}$. 
Führt man die Hydrolyse in einem Natriumacetatpuffer bei pH 4.5 und mit Lipasen durch, können in allen Fällen sowohl die Cyanhydrinester als auch die freien Cyanhydrine in optisch aktiver Form erhalten werden ${ }^{[41]}$. Für die Lipase aus Pseudomonas fluorescens (A), die Lipase PS (B) und die Lipase P (C) ist dabei jeweils das (S)-Acetat das Substrat, so daB (S)-Cyanhydrine resultieren. Die Lipase aus Candida cylindracea (D) und die Lipase AY (E) katalysieren dagegen die Bildung der $(R)$-Cyanhydrine (Schema 4) ${ }^{[41]}$. Für die Lipasen $\mathbf{A}, \mathbf{B}$ und $\mathbf{C}$ sind

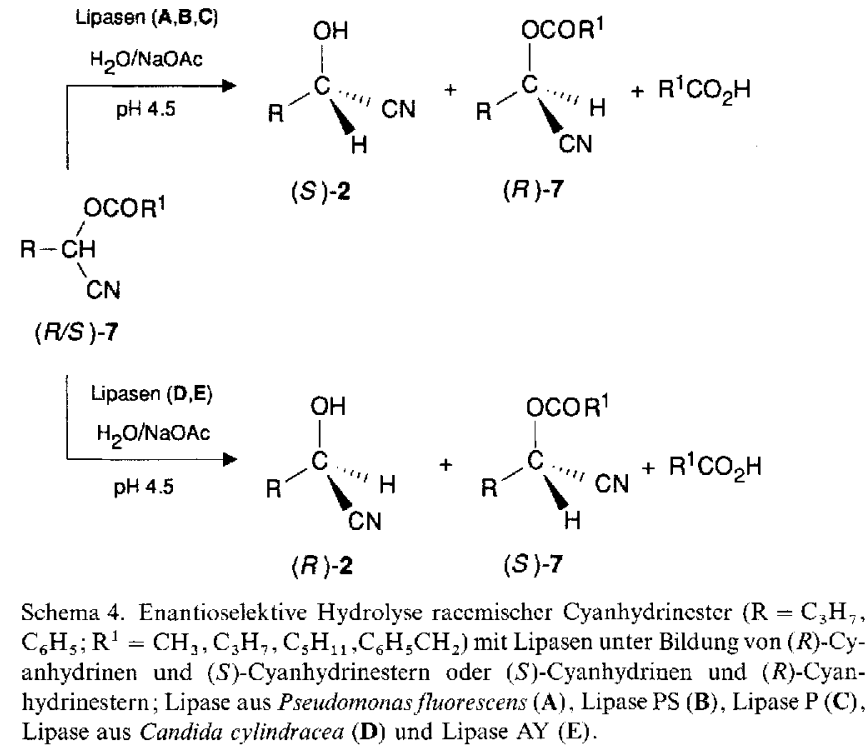

aromatische Cyanhydrinester $\left(\mathrm{R}=\mathrm{C}_{6} \mathrm{H}_{5}\right)$ gute Substrate, nicht jedoch die aliphatischen Vertreter $\left(\mathrm{R}=\mathrm{C}_{3} \mathrm{H}_{7}\right)$. Die höchsten optischen Ausbeuten an $(S)$-Cyanhydrinen (bis $97 \% e e$ ) werden dabei mit den Butyraten $\left(\mathrm{R}^{1}=\mathrm{C}_{3} \mathrm{H}_{7}\right)$ erreicht. Umgekehrt sind für die Lipasen $\mathbf{D}$ und $\mathbf{E}$ aliphatische Cyanhydrinester $\left(\mathrm{R}=\mathrm{C}_{3} \mathrm{H}_{7}\right)$ gute Substrate, wobei auch in diesen Fällen die Butyrate $\left(\mathrm{R}^{1}=\mathrm{C}_{3} \mathrm{H}_{7}\right)$ die besten optischen Ausbeuten an $(R)$ Cyanhydrinen (bis $86 \%$ ee) ergeben.

\subsection{Enantioselektive Veresterung racemischer Cyanhydrine}

Chirale Cyanhydrine racemisieren in organischen Lösungsmitteln nicht merklich. Eine durch Lipasen katalysierte enantioselektive Veresterung racemischer Cyanhydrine in einem organischen Lösungsmittel ist deshalb für eine Racematspaltung, bei der dic ungeschützten optisch aktiven Cyanhydrine hergestellt werden sollen, besonders geeignet ${ }^{[41,43]}$. Das beste Acylierungsreagens ist hierbei das irreversibel reagierende Vinylacetat ${ }^{[44]}$, und das am besten geeignete Lösungsmittel ist Dichlormethan ${ }^{[41}$. Unter diesen Acylierungsbedingungen weisen die verwendeten Lipasen A-D annähernd die gleiche Konfigurationsspezifität auf wie bei der in Abschnitt 6.1. beschriebenen enantioselektiven Hydrolyse racemischer Cyanhydrinester (Schema 5).

Die höchsten Enantiomerenüberschüsse an $(S)$-Acetaten $(S)$ 7 (bis $98 \%$ ee) wurden bei aromatischen Cyanhydrinen mit der Pseudomonas-fluorescens-Lipase (A) erhalten ${ }^{[41]}$. Aber auch die

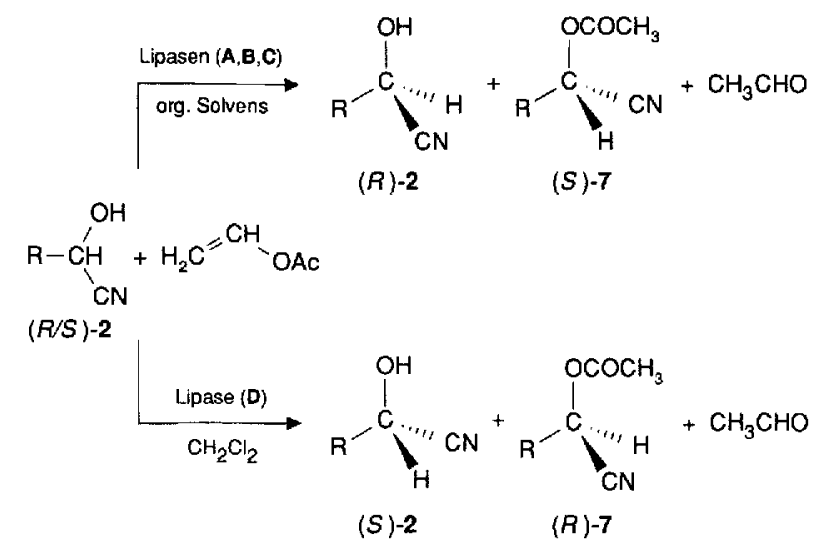

Schema 5. Enantioselektive Veresterung racemischer Cyanhydrine $\left(\mathrm{R}=\mathrm{C}_{3} \mathrm{H}_{7}\right.$, $\mathrm{C}_{6} \mathrm{H}_{5}, \mathrm{C}_{6} \mathrm{H}_{5} \mathrm{CH}_{2} \mathrm{CH}_{2}, 4-\mathrm{HOC}_{6} \mathrm{H}_{4}, 3-\mathrm{CH}_{3} \mathrm{OC}_{6} \mathrm{H}_{4}$ etc.) mit Vinylacetat in organischen lösungsmitteln mit Lipasen (vgl. Schema 4).

Acylierung aliphatischer Cyanhydrine unter Bildung der Acetate (S)-7 wird durch die Lipase A katalysiert.

Ein entscheidender Nachteil kinetischer Racematspaltungen besteht generell darin, daß höchstens $50 \%$ des erwünschten Enantiomers aus einem Racemat erhalten werden können. Für praktische Anwendungen ist es in der Regel notwendig, in einer anschließenden Reaktion das unerwünschte Enantiomer zu racemisieren und das Racemat erneut der Trennung zuzuführen. Bei der Trennung racemischer Cyanhydrine durch enantioselektive Acetylierung mit Isopropenylacetat konnte eine nachgeschaltete, getrennte Racemisierung dadurch umgangen werden, daß in einem organischen Lösungsmittel in Gegenwart eines basischen Ionenaustauschers acetyliert wurde, wobei die Base zwar zur Racemisierung der Cyanhydrine ausreicht, die gebildeten optisch aktiven Cyanhydrinacetate jedoch nicht racemisiert werden ${ }^{[45]}$. So war es möglich, das eingesetzte Racemat vollständig in ein Enantiomer zu überführen. Die dann zur Gewinnung der Cyanhydrine selbst noch erforderliche Hydrolyse der anfallenden enantiomerenreinen Cyanhydrinacetate ist racemisierungsfrei möglich.

Neben der enantioselektiven Verseifung racemischer Cyanhydrinester (vgl. Abschnitt 6.1.) und der enantioselektiven Veresterung racemischer Cyanhydrine (vgl. Abschnitt 6.2.) kann eine Trennung racemischer Cyanhydrinester auch durch eine Lipase-katalysierte Umesterung mit höher siedenden Alkoholen in organischen Lösungsmitteln erreicht werden ${ }^{[41,46]}$.

\section{Stereoselektive Reaktionen optisch aktiver Cyanhydrine}

Da Cyanhydrine als $\alpha$-substituierte Carbonsäurederivate ein großes Synthesepotential haben, eröffnet sich damit die Möglichkeit, über stereoselektive Folgereaktionen chiraler Cyanhydrine eine Reihe anderer wichtiger Verbindungsklassen mit Asymmetriezentren zu erschließen. Bei den Reaktionen der Cyanhydrine kann zwischen zwei Typen unterschieden werden: zum einen Reaktionen der Nitrilgruppe unter Erhalt der $\mathrm{OH}-$ Funktion und zum anderen Reaktionen der Hydroxygruppe. Für beide Reaktionstypen muß für Anwendungen in der Synthese ein racemisierungsfreier Verlauf gewährleistet sein. 


\subsection{Reaktionen der Nitrilgruppe}

Im Vergleich mit $\alpha$-Aminosäuren kommen nur wenige optisch aktive $\alpha$-Hydroxycarbonsäuren in der Natur vor. Es mußten deshalb allgemeine Verfahren zu ihrer Synthese entwickelt werden. Heute sind chirale $\alpha$-Hydroxycarbonsäuren chemisch ${ }^{[47]}$, fermentativ ${ }^{[48]}$ oder enzymatisch ${ }^{[49,50]}$ zugänglich. Ein häufig angewandtes Verfahren zur Gewinnung spezieller $(S)-\alpha$ - $\mathrm{Hy}$ droxycarbonsäuren ist die Diazotierung natürlich vorkommender L- $x$-Aminosäuren in wäßrigem. Medium. Die primär gebildeten Diazoniumsalze reagieren dabei crst intramolekular unter Konfigurationsumkehr zu $\alpha$-Lactonen, die dann unter Inversion mit Wasser als Nucleophil zu $\alpha$-Hydroxycarbonsäuren der gleichen Konfiguration wie die der eingesetzten Aminosäuren hydrolysieren. Durch Aktivierung der OH-Gruppe der $\alpha$-Hydroxycarbonsäuren und anschließende Umsetzung mit Sauerstoffnucleophilen können die ( $S$ )-2-Hydroxycarbonsäuren unter Konfigurationsumkehr in die $(R)$-Enantiomere überführt werden ${ }^{[51]}$.

Ein bezüglich der möglichen breiten Substratvariation äußerst interessanter, allgemeiner Zugang zu $(R)$ - und $(S)$-2-Hydroxycarbonsäuren bot sich in der Hydrolyse chiraler Cyanhydrine an, wenn sich diese racemisierungsfrei durchführen läßt. Die Hydrolyse eines optisch aktiven Cyanhydrins mit konzentrierter Salzsäure wurde erstmals für Mandelsäurenitril beschrieben $^{\left[1 \mathrm{Iaj}^{\mathrm{a}}\right.}$ und diente hier der Bestimmung der optischen Reinheit des Nitrils. In späteren Arbeiten wurde jedoch angenommen, daß die säurekatalysierte Hydrolyse ungeschützter Cyanhydrine in einigen Fällen unter teilweiser Racemisierung erfolgt ${ }^{\left[31,3 s^{a}\right]}$. Sauerstoffgeschützte Cyanhydrine werden dagegen sowohl im sauren ${ }^{[521}$ als auch im basischen ${ }^{[44 a]}$ Medium weitgehend unter Erhaltung der Konfiguration zu $\alpha$-Hydroxycarbonsäuren hydrolysiert. Da sowohl die Bildung als auch die Spaltung der Cyanhydrine in Aldehyd und Blausäure basenkatalysiert ist, konnte nur bei einer mit Säuren katalysierten Hydrolyse ungeschützter Cyanhydrine mit einem racemisierungsfreien Verlauf gerechnet werden.

$(R)$ - und $(S)$-Aldehyd-Cyanhydrine ${ }^{[13 e, 23]}$ sowie $(R)$-KetonCyanhydrine ${ }^{[33]}$ lassen sich mit konzentrierter Salzsäure racemisierungsfrei, unter vollständiger Erhaltung der Konfiguration, und in sehr guten Ausbeuten zu den entsprechenden $(R)$ - bzw. $(S)$-Hydroxycarbonsäuren hydrolysieren (Schema 6, 7, Tabelle 6,7$)$.

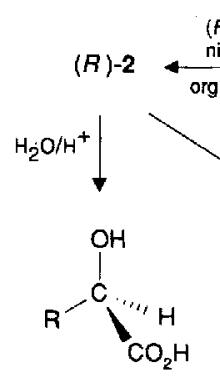

(R)-8

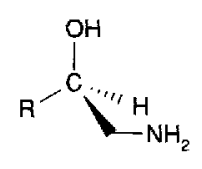

(R) -9

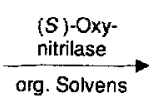

(S)-2

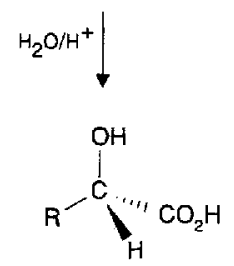

(S)-8
Schema 6. Säurekatalysierte Hydrolyse von $(R)$ - und $(S)$-Cyanhydrinen zu $(R)$ bzw. (S)-2-Hydroxycarbonsäuren sowie racemisierungsfreie Hydrierung von $(R)$ Cyanhydrinen zu $(R)$-2-Aminoalkoholen.
Tabelle 6. Säurekatalysierte Hydrolyse von in situ durch enzymkatalysierte Additionen von $H C N$ an Aldehyde 1 erhaltenen $(R)$ - und $(S)$-Cyanhydrinen 2 [a] unter Bildung von $(R)$ - bzw. $(S)-\alpha-$ Hydroxycarbonsäuren 8.

\begin{tabular}{lllllll}
\hline $\mathrm{R}$ & $(R)-2$ & $(S)-2$ & \multicolumn{3}{c}{$(R)-8$} & \multicolumn{2}{c}{$(S)-8$} \\
& $e e$ & $e e$ & Ausb. & $e e[\mathrm{~b}]$ & Ausb. & ee $[\mathrm{b}]$ \\
& {$[\%]$} & {$[\%]$} & {$[\%]$} & {$[\%]$} & {$[\%]$} & {$[\%]$} \\
\hline $\mathrm{C}_{6} \mathrm{H}_{5}$ & 99 & 97 & 82 & 99 & 77 & 98 \\
$4-\mathrm{H}_{3} \mathrm{CC}_{6} \mathrm{H}_{4}$ & 98 & 78 & 82 & 98 & 75 & 78 \\
$4-\mathrm{ClC}_{6} \mathrm{H}_{4}$ & 99 & 54 & 71 & 99 & 63 & 54 \\
$3-\mathrm{C}_{6} \mathrm{H}_{5} \mathrm{OC}_{6} \mathrm{H}_{4}$ & 97 & 96 & 72 & 97 & 70 & 96 \\
$3-\mathrm{O}_{2} \mathrm{NC}_{6} \mathrm{H}_{4}$ & 89 & - & 81 & 87 & - & - \\
$\mathrm{C}_{6} \mathrm{H}_{5} \mathrm{CH}_{2}$ & 74 & & 90 & 74 & - & - \\
$c-\mathrm{C}_{6} \mathrm{H}_{11}$ & 99 & & 88 & 97 & & - \\
$3-\mathrm{BrC}_{6} \mathrm{H}_{4}$ & - & 92 & - & - & 76 & 90 \\
$3-\mathrm{ClC}_{6} \mathrm{H}_{4}$ & - & 91 & - & - & 69 & 91 \\
$3-\mathrm{H}_{3} \mathrm{COC}_{6} \mathrm{H}_{4}$ & - & 89 & - & - & 67 & 90 \\
$\mathrm{H}_{3} \mathrm{C}_{2}\left(\mathrm{CH}_{2}\right)_{2}$ & 96 & - & 85 & 96 & - & - \\
$2-\mathrm{H}_{3} \mathrm{CS}_{2}\left(\mathrm{CH}_{2}\right)_{2}$ & 98 & - & 87 & 98 & - & - \\
-
\end{tabular}

[a] Rohprodukte. [b] Nach Umsetzung mit HCl-gesättigtem Isopropylalkohol zu den Isopropylestern und anschlie[3ender Derivatisierung zu den diastereomeren $(R)$ MTPA-Estern gaschromatographisch bestimmt $[13,23]$.

$$
\begin{aligned}
& 3,4+\mathrm{HCN} \stackrel{\substack{(R)-O x y-\\
\text { nitrilase }}}{\longrightarrow}(R)-5,(R)-6 \stackrel{\mathrm{H}_{2} \mathrm{O}^{+}}{\longrightarrow} \\
& (R)-10: \mathrm{R}^{1}=\text { Alkyl, } \mathrm{R}^{2}=\mathrm{CH}_{3} \\
& (R)-11: \mathrm{R}^{\dagger}=\text { Alkyl, } \mathrm{R}^{2}=\mathrm{C}_{2} \mathrm{H}_{5}
\end{aligned}
$$

Schema 7. Säurekatalysierte Hydrolyse von $(R)$-Keton-Cyanhydrinen zu $(R)$-Hydroxycarbonsāuren.

\begin{tabular}{|c|c|c|c|c|}
\hline \multirow[t]{2}{*}{$\mathrm{R}^{1}$} & \multirow[t]{2}{*}{$\mathrm{R}^{2}$} & \multirow{2}{*}{$\begin{array}{l}(R)-5,(R)-6 \\
e e[\%]\end{array}$} & \multicolumn{2}{|c|}{$(R)-10,(R)-11$} \\
\hline & & & Ausb. $[\%]$ & $e e[\mathrm{a}][\%]$ \\
\hline $\mathrm{C}_{2} \mathrm{H}_{5}$ & $\mathrm{CH}_{3}$ & 76 & 85 & 76 \\
\hline$n \mathrm{C}_{3} \mathrm{H}_{7}$ & $\mathrm{CH}_{3}$ & 97 & 64 & 99 \\
\hline$n \mathrm{C}_{4} \mathrm{H}_{9}$ & $\mathrm{CH}_{3}$ & 98 & 72 & 98 \\
\hline$n \mathrm{C}_{5} \mathrm{H}_{11}$ & $\mathrm{CH}_{3}$ & 98 & 78 & 98 \\
\hline$\left(\mathrm{H}_{3} \mathrm{C}\right)_{2} \mathrm{CH}$ & $\mathrm{CH}_{3}$ & 90 & 76 & 90 \\
\hline$\left(\mathrm{H}_{3} \mathrm{C}\right)_{2} \mathrm{CHCH}_{2}$ & $\mathrm{CH}_{3}$ & 98 & 89 & 96 \\
\hline$\left(\mathrm{H}_{3} \mathrm{C}\right)_{2} \mathrm{CH}\left(\mathrm{CH}_{2}\right)_{2}$ & $\mathrm{CH}_{3}$ & 98 & 88 & 99 \\
\hline$n \mathrm{C}_{3} \mathrm{H}_{7}$ & $\mathrm{C}_{2} \mathrm{H}_{5}$ & 85 & 82 & 85 \\
\hline$n \mathrm{C}_{4} \mathrm{H}_{9}$ & $\mathrm{C}_{2} \mathrm{H}_{5}$ & 90 & 89 & 90 \\
\hline
\end{tabular}

Tabelle 7. Säurekatalysierte Hydrolyse von in situ durch enzymkatalysierte Additionen von HCN an Ketone 3 und 4 erhaltenen $(R)-K e t o n-C y a n h y d r i n e n(R)-5$ bzw. (R)-6 unter Bildung von $\alpha$-Hydroxycarbonsäuren $(R)-10$ bzw. (R)-11 [33].

[a] Nach Veresterung mit Diazomethan auf $\beta$-Cyclodextrin-Säulen gaschromatographisch bestimmt [33].

Die praktische Durchführung der Hydrolyse zu $\alpha$-Hydroxycarbonsäuren ist dabei sehr einfach: Zunächst werden die optisch aktiven Cyanhydrine enzymkatalysiert aus den Carbonylverbindungen und Blausäure in einem organischen Lösungsmittel hergestellt. Nach Abfiltrieren des auf Avicel fixierten Enzyms wird das Lösungsmittel abdestilliert und die als Rückstand verbleibenden Cyanhydrine ohne weitere Reinigung mit konzentrierter Salzsäure hydrolysiert. Nach dem Entfernen der Salzsäure werden die chiralen $\alpha$-Hydroxycarbonsäuren mit Diethylether extrahiert, isoliert und charakterisiert. Ein Vergleich der optischen Reinheiten der Cyanhydrin-Rohprodukte mit denen der isolierten $\alpha$-Hydroxycarbonsäuren zeigt, da $\beta$ in allen untersuchten Fällen bei der Hydrolyse keine Racemisierung auftritt (Tabelle 6,7$)$. 
Von den Methoden zur Synthese chiraler $\alpha$-Hydroxycarbonsäuren ist die säurekatalysierte Hydrolyse optisch aktiver Cyanhydrine wegen des einfachen Zugangs zu strukturell sehr unterschiedlichen Cyanhydrinen und deren problemloser racemisierungsfreier Verseifung die universellste.

Optisch aktive $\alpha$-Hydroxyaldehyde sind als Strukturelement in Naturstoffen und als Ausgangsverbindungen für asymmetrische Synthesen ebenfalls von großer Bedeutung ${ }^{[52]}$. Eine direkte Umwandlung optisch aktiver Cyanhydrine in die entsprechenden $\alpha$-Hydroxyaldehyde wäre ein sehr einfacher Zugang zu dieser Verbindungsklasse. Tatsächlich wurden racemische Cyanhydrine mit Raney-Nickel in saurer Lösung direkt zu $\alpha$-Hydroxyaldehyden hydriert, deren Isolierung allerdings Schwierigkeiten bereitet $^{[53 a]}$. Bei der Übertragung dieses Verfahrens auf optisch aktive Cyanhydrine wurde aus der Konfigurationsbestimmung der Folgeprodukte geschlossen, da 3 die Hydrierung weitgehend racemisierungsfrei verläuft ${ }^{[53 b]}$. Wegen der mäßigen Ausbeuten und der Schwierigkeiten bei der Produktisolierung, ist für die Synthese chiraler $\alpha$-Hydroxyaldehyde aus Cyanhydrinen der Weg über die leicht zugänglichen $\alpha$-Hydroxycarbonsäuren und deren selektive Hydrierung einer direkten Hydrierung der Cyanhydrine jedoch vorzuziehen ${ }^{\text {[52d, 54]. }}$.

Die Hydrierung zu 2-Aminoalkoholen ist ebenfalls von großem praktischen Interesse, da sich 2-Aminoalkohole durch vielseitige biologische Aktivitäten auszeichnen ${ }^{[55]}$. Bei den als Wirkstoffe wichtigen 2-Aminoalkoholen kann zwischen Verbindungen vom Adrenalin-Typ einerseits, mit der Aminogruppe an einem primären Kohlenstoff, und Verbindungen vom Ephedrin-Typ andererseits, mit der Aminogruppe an einem sekundären Kohlenstoff, unterschieden werden. Verbindungen vom Adrenalin-Typ können durch Hydrierung $O$-geschützter chiraler Cyanhydrine mit unterschiedlichen Hydrierungsmitteln erhalten werden, wobei keine nachweisbare Racemisierung auftritt ${ }^{[4 a, 43 a, 56]}$. Erstaunlicherweise lassen sich aber auch ungeschützte chirale Cyanhydrine mit $\mathrm{LiAlH}_{4}$ racemisierungsfrei zu Aminoalkoholen hydrieren $^{[13 c]}$. Hierbei können wie bei der Synthese der $\alpha$-Hydroxycarbonsäuren die Rohprodukte der in organischen Lösungsmitteln anfallenden chiralen Cyanhydrine direkt hydriert werden (Schema 6 , Tabelle 8$)^{\mid 130\rceil}$.

Tabelle 8. Hydrierung von in situ durch enzymkatalysierte Addition von $\mathrm{HCN}$ an Aldebyde 1 erhaltenen $(R)$-Cyanhydrinen $(R)$ - 2 mit Lithiumaluminiumbydrid unter Bildung von Aminoalkoholen $(R)-9[13 \mathrm{c}]$

\begin{tabular}{|c|c|c|c|c|c|c|c|}
\hline $\mathrm{R}$ & $\begin{array}{l}(R)-2 \\
e e \\
{[\%]}\end{array}$ & \multicolumn{2}{|c|}{$\begin{array}{l}\quad(R)-9 \\
\text { Ausb. ee }[a] \\
{[\%] \quad[\%]}\end{array}$} & $\mathrm{R}$ & $\begin{array}{l}(R)-2 \\
e e^{\circ} \\
{[\%]}\end{array}$ & $\begin{array}{l}\quad(R) \\
\text { Ausb. } \\
{[\%]}\end{array}$ & $\begin{array}{l}-9 \\
\text { ee }[\mathrm{a}] \\
{[\%]}\end{array}$ \\
\hline $\mathrm{C}_{6} \mathrm{H}_{5}$ & 99 & 92 & 99 & $2-\mathrm{H}_{3} \mathrm{CS}\left(\mathrm{CH}_{2}\right)_{2}$ & 98 & 31 & 98 \\
\hline $3-\mathrm{C}_{6} \mathrm{H}_{5} \mathrm{OC}_{6} \mathrm{H}_{4}$ & 98 & 97 & 95 & $n \mathrm{C}_{3} \mathrm{H}_{7}$ & 96 & 99 & 89 \\
\hline $4-\mathrm{H}_{3} \mathrm{CC}_{6} \mathrm{H}_{4}$ & 99 & 100 & $>98$ & 2-Furyl & 98 & 71 & 98 \\
\hline$c-\mathrm{C}_{6} \mathrm{H}_{11}$ & 91 & 94 & 80 & $\left(\mathrm{H}_{3} \mathrm{C}\right)_{3} \mathrm{C}$ & 93 & 70 & $>90$ \\
\hline
\end{tabular}

[a] Nach Umsetzung mit Trifluoressigsäure zu den entsprechenden $(R)-\beta-N, O$-Bis(trifluoracetyl)aminoalkoholen gaschromatographisch auf Chirasil-Val-Säulen bestimmt $[13 \mathrm{c}]$.

Die Synthese von 2-Aminoalkoholen vom Ephedrin-Typ ausgehend von Cyanhydrinen gelang erstmals Krepski et al., indem sie O-geschützte racemische Cyanhydrine mit Grignard-Verbindungen umsetzten und die so erhaltenen Imine mit $\mathrm{NaBH}_{4}$ hydrierten ${ }^{[57]}$. Diese Methode konnte später erfolgreich auf $(R)$ - und $(S)$-Cyanhydrine angewendet werden ${ }^{[58,59]}$. Die Untersuchung des stereochemischen Verlaufs ergab, daß alle Reaktionen sowohl der $(R)$ - als auch der $(S)$-Cyanhydrine racemisierungsfrei bezüglich der Konfiguration an $\mathrm{C}-1$ ablaufen und da $B$ die Hydrierung an C-2 mit hoher Diastereoselektivität erfolgt ${ }^{[59]}$. Über einen Chelat-Komplex kontrolliert entstehen dabei nahezu ausschließlich erythro-Verbindungen (Schema 8 , Tabelle 9) ${ }^{[59]}$. Überwiegend threo-Verbindungen werden erhal-
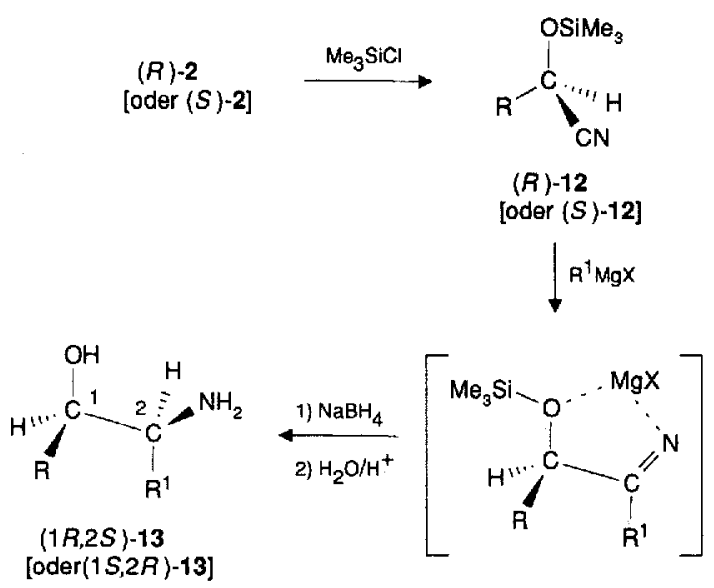

Schema 8. Stereoselektive Synthese von 2-Aminoalkoholen. $\mathrm{R}=\mathrm{C}_{3} \mathrm{H}_{7}, \mathrm{C}_{6} \mathrm{H}_{5}$; $\mathrm{R}^{1}=\mathrm{CH}_{3}, \mathrm{C}_{2} \mathrm{H}_{5}, \mathrm{C}_{6} \mathrm{H}_{5} ; \mathrm{X}=\mathrm{Br}, \mathrm{I}$.

Tabelle 9. Synthese der 2-Aminoalkohole $(1 R, 2 S)$ - und $(1 S, 2 R)$-13 aus den Cyanhydrinen $(R)$ - bzw. ( $S$ )-2 (Rohprodukte) durch Bildung der Trimethylsilylether $(R)$ bzw. $(S)$-12, deren Umsetzung mit Grignard-Verbindungen und anschließende $\mathrm{Hy}$ drierung mit Natriumborhydrid [59].

\begin{tabular}{|c|c|c|c|c|c|c|c|c|}
\hline \multirow[t]{2}{*}{$\mathbf{R}$} & \multicolumn{2}{|c|}{ Cyanhydrin 2} & \multicolumn{6}{|c|}{ 2-Aminoalkohol $(1 R, 2 S)$ - oder $(1 S, 2 R)-13$} \\
\hline & $\begin{array}{l}e e \\
{[\%]}\end{array}$ & $\begin{array}{l}\text { Konfigu- } \\
\text { ration }\end{array}$ & $\mathrm{R}^{1}$ & $\begin{array}{l}\text { Ausb. [a] } \\
{[\%]}\end{array}$ & $\begin{array}{l}e e[b] \\
{[\%]}\end{array}$ & $\begin{array}{l}d e[\mathrm{~b}] \\
{[\%]}\end{array}$ & $\begin{array}{l}e e[c] \\
{[\%]}\end{array}$ & $\begin{array}{l}d e[\mathrm{c}] \\
{[\%]}\end{array}$ \\
\hline $\mathrm{C}_{6} \mathrm{H}_{5}$ & $>99$ & $(R)$ & $\mathrm{C}_{6} \mathrm{H}_{5}$ & 83 & 99 & 99 & 100 & 100 \\
\hline $\mathrm{C}_{6} \mathrm{H}_{5}$ & $>99$ & $(R)$ & $\mathrm{C}_{2} \mathrm{H}_{5}$ & 53 & 97 & 94 & $>99$ & $>99$ \\
\hline $\mathrm{C}_{6} \mathrm{H}_{5}$ & $>99$ & $(R)$ & $\mathrm{CH}_{3}$ & 50 & 99 & 92 & 99 & $>99$ \\
\hline $\mathrm{C}_{3} \mathrm{H}_{7}$ & 95 & $(R)$ & $\mathrm{C}_{6} \mathrm{H}_{5}$ & 93 & 92 & 93 & $>99$ & $>99$ \\
\hline $\mathrm{C}_{3} \mathrm{H}_{7}$ & 95 & $(R)$ & $\mathrm{CH}_{3}$ & 44 & 93 & 63 & - & 63 \\
\hline $\mathrm{C}_{6} \mathrm{H}_{5}$ & $>99$ & (S) & $\mathrm{C}_{6} \mathrm{H}_{5}$ & 95 & 99 & 99 & 100 & 100 \\
\hline $\mathrm{C}_{6} \mathrm{H}_{5}$ & $>99$ & $(S)$ & $\mathrm{CH}_{3}$ & 66 & 99 & 90 & $>99$ & $>99$ \\
\hline
\end{tabular}

[a] Als Hydrochloride. [b] de- und ee-Werte nach Umsetzung der Rohprodukte mit Pivalylchlorid und Filtration durch Kieselgel gaschromatographisch bestimmt. [c] de- und ee-Werte nach einmaligem Umkristallisieren.

ten, wenn an das $O$-geschützte Cyanhydrin zuerst ein komplexes Hydrid ( $N$-Diisobutylaluminiumhydrid) und anschließend eine Organolithiumverbindung addiert wird ${ }^{[60]}$. Bei diesem Verfahren werden allerdings deutlich schlechtere Diastereoselektivitäten gefunden ${ }^{[60]}$.

Bei den durch Hydrierung ${ }^{[61 \mathrm{a}]}$ oder durch Addition von Grignard-Reagentien ${ }^{[62 b]}$ an $\mathrm{O}$-geschützte Cyanhydrine primär entstehenden Iminen ist eine Transiminierung mit primären Aminen möglich, was vorteilhaft für die Synthese der biologisch interessanten N-substituierten Aminoalkohole sein kann. Auch Blausäure kann an die Imine addiert werden, wobei ausgehend von chiralen Cyanhydrinen $\beta$-Hydroxy- $\alpha$-aminosäuren stereoselektiv zugänglich sind ${ }^{[62]}$. Die Hydrolyse der durch Addition 
von Grignard-Verbindungen an O-geschützte, chirale Cyanhydrine entstehenden Imine führt zu optisch aktiven Acyloinen $^{[18 a, 63]}$.

\subsection{Reaktionen der Hydroxygruppe}

Eine wichtige Erweiterung des Synthesepotentials optisch aktiver Cyanhydrine ist die Umwandlung der $\mathrm{OH}$-Funktion in eine gute Abgangsgruppe, die dann durch Nucleophile stereoselektiv substituiert werden kann ${ }^{[64]}$.

Die nucleophile Substitution aktivierter $\alpha$-Hydroxycarbonsäuren und deren Ester bereitet wenig Schwierigkeiten ${ }^{[1 \mathrm{ja}, 65]}$, über entsprechende Reaktionen $\alpha$-substituierter Nitrile ist hingegen wenig bekannt ${ }^{[66]}$. Von optisch aktiven Nitrilen mit $\alpha$-ständiger nucleofuger Abgangsgruppe sind bisher lediglich $\alpha$-Halogennitrile hergestellt und beschrieben worden ${ }^{[66]}$. Bei der nucleophilen Substitution dieser Verbindungen werden Halogenid-Ionen frei, die selbst als gute Nucleophile reagieren, so $\mathrm{da} \beta$ die $\alpha$-Halogennitrile unter diesen Bedingungen teilweise racemisieren ${ }^{[66]}$. Wesentlich konfigurationsstabiler als $\alpha$ Halogennitrile sind die aus chiralen Cyanhydrinen $(R)-2$ durch Sulfonierung einfach zugänglichen optisch aktiven $\alpha$-Sulfonyloxynitrile $(R)-14^{[64]}$ (Schema 9) . $\alpha$-Sulfonyloxynitrile, die sich von aliphatischen Cyanhydrinen ableiten, sind dabei deutlich konfigurationsstabiler als die sulfonylaktivierten aromatischen Cyanhydrine ${ }^{[64]}$. Auch bezüglich der Stereoselektivität der Reaktionen mit Nucleophilen bestehen deutliche Unterschiede. Während aliphatische $\alpha$-Sulfonyloxynitrile $(R)$-14 unter vollständiger Konfigurationsumkehr unter milden Bedingungen mit $\mathrm{Nu}$ cleophilen reagieren, tritt bei Umsetzungen der aromatischen Vertreter mit schwachen Nucleophilen teilweise Racemisierung auf $^{[64]}$

Über die Substitution optisch aktiver $\alpha$-Sulfonyloxynitrile $(R)$ 14 mit Nucleophilen sind viele Verbindungen, z.B. $\alpha$-Azidonitrile $(S)$-15, $\alpha$-Aminonitrile $(S)$-16, $\alpha$-Aminosäuren $(S)$-17 und Aziridine $(S)-18$, in optisch reiner Form zugänglich ${ }^{[641}$ (Schema 9). Die Konfigurationsumkehr bei der Reaktion der stabilen $(R)-2$ Sulfonyloxynitrile $(R)-14$ mit Acetaten ${ }^{[64 b]}$ unter Bildung von Cyanhydrinestern (S)-19 ermöglicht auch die Synthese der (S)konfigurierten aliphatischen Cyanhydrine $(S)$-2, die über eine durch $(S)$-Oxynitrilase katalysierte Addition von Blausäure an Aldehyde nicht zugänglich sind ${ }^{[23,24]}$.

Die Sulfonylaktivierung von Cyanhydrinen und ihre Nutzung für die Synthese ist sehr allgemein anwendbar, da sowohl das Cyanhydrin als auch das Nucleophil variiert werden kann. Daneben sind jedoch auch direkte stereoselektive Reaktionen der OH-Gruppe in Cyanhydrinen mit speziellen Reagentien möglich. So können die $(S)$-2-Fluornitrile $(S)$-20 aus den $(R)$-Cyanhydrinen $(R)-2$ durch Umsetzung mit Diethylaminoschwefeltrifluorid (DAST) in guten optischen Ausbeuten erhalten werden ${ }^{[67]}$. Die $\alpha$-Fluornitrile $(S)$-20 lassen sich racemisierungsfrei zu den $\alpha$-Fluorcarbonsäuren $(S)$-21 hydrolysieren ${ }^{[67]}$ (Schema 10).

Ein weiteres Beispiel für eine stereoselektive Reaktion in diesem Zusammenhang ist die Mitsunobu-Reaktion mit optisch aktiven Cyanhydrinen in Gegenwart von Carbonsäuren, wodurch unter Konfigurationsumkehr auch auf diesem Weg aus den $(R)$-Cyanhydrinen die $(S)$-Enantiomere zugänglich sind ${ }^{[68]}$.
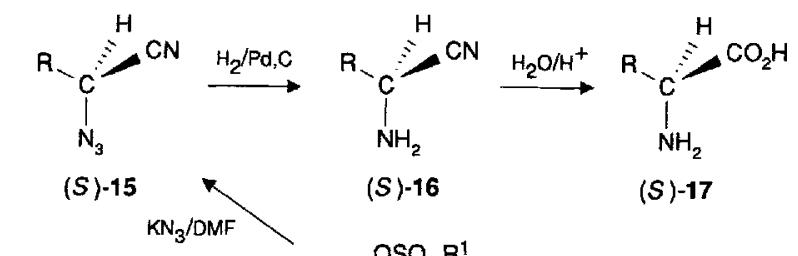

(S)-16

(S)-17

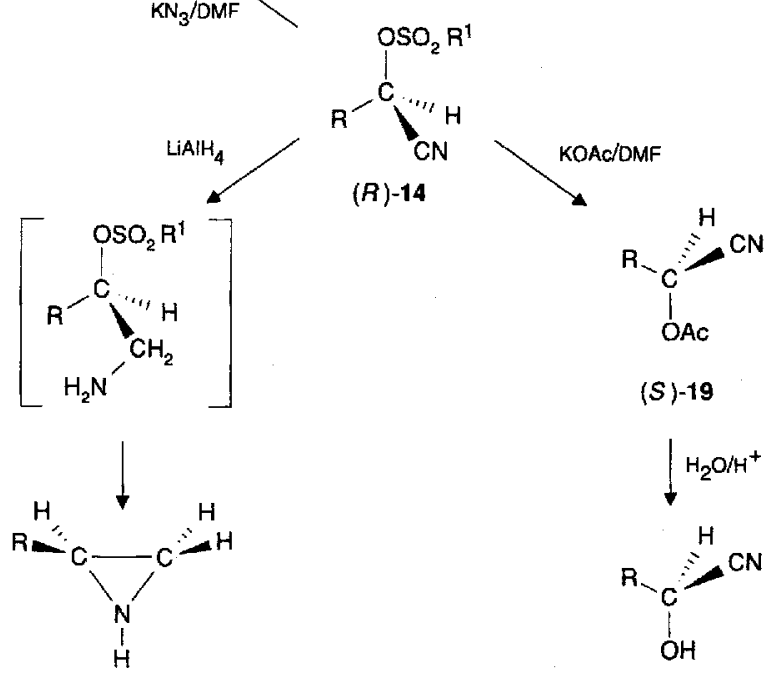

(S)-18

$(S)-2$

Schema 9. Stereoselektive Umsetzungen von Sulfonyl-aktivierten $(R)$-Cyanhydrinen mit Nucleophilen unter Konfigurationsumkehr. $\mathrm{R}^{1}=\mathrm{CH}_{3}, \mathrm{CF}_{3}, 4-\mathrm{CH}_{3} \mathrm{C}_{6} \mathrm{H}_{4}$

$($ ( ) -2

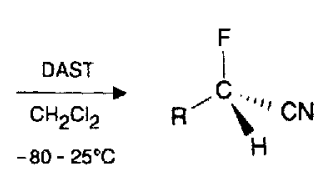

(S) -20

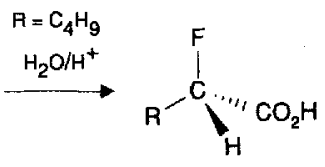

(S)-21

$$
\mathrm{R}=\mathrm{C}_{3} \mathrm{H}_{7}, \mathrm{C}_{4} \mathrm{H}_{9},\left\langle\mathrm{H}_{3} \mathrm{C}\right)_{2} \mathrm{CHCH}_{2}, c-\mathrm{C}_{6} \mathrm{H}_{11}, \mathrm{C}_{6} \mathrm{H}_{5}
$$

Schema 10. Synthese von $(S)$-2-Fluornitrilen und $(S)$-2-Fluorcarbonsätren aus (R)-Cyanhydrinen.

\section{Zusammenfassung und Ausblick}

Obwohl die enzymkatalysierte asymmetrische Synthese von Cyanhydrinen schon fast neunzig Jahre bekannt ist, wurde sie erst vor weniger als zehn Jahren für die organische Synthese bedeutungsvoll. Der entscheidende Durchbruch war eine deutliche Verbesserung der Enantiomerenüberschüsse durch Unterdrückung der chemischen Cyanhydrinbildung. Dies gelang durch die Verwendung organischer Lösungsmittel als Reaktionsmedium und durch das Arbeiten bei niedrigen $\mathrm{pH}$-Werten.

Nachdem insbesondere $(R)$-Cyanhydrine auf diese Weise leicht zugänglich geworden waren, wurde in wenigen Jahren das große Synthesepotential, das diese Verbindungen als $\alpha$-substituierte Carbonsäurederivate haben, erschlossen. Da die Folgereaktionen optisch aktiver Cyanhydrine so geführt werden können, daß sie unter Erhaltung des Chiralitätszentrums und beim Aufbau weiterer Asymmetriezentren weitgehend diastereoselektiv verlaufen, wird über chirale Cyanhydrine eine Vielzahl anderer wichtiger Verbindungsklassen mit Asymmetriezentren zugänglich. Besondere Bedeutung kommt dem Synthesepotential chiraler Cyanhydrine unter anderem bei der Synthese von Wirkstoffen mit 
Chiralitätszentren zu. Selbst wenn solche Wirkstofle, z.B. 2Aminoalkohole, heute noch in racemischer Form in den Handel kommen, ist für ihre Zulassung die Synthese aller Stereoisomere erforderlich, um das Wirkungsspektrum und den Metabolismus für jedes Stereoisomer getrennt aufzuklären. Chirale Cyanhydrine eröffnen dabei in vielen Fällen einen einfachen Zugang zu den einzelnen Stereoisomeren.

Während die $(R)$-Oxynitrilase aus Bittermandeln cin breites Spektrum von Substraten akzeptiert und auch in technisch relevanten Mengen problemlos erhalten werden kann, ist für die (S)-Oxynitrilase aus Sorghum das Substratspektrum weitgehend auf aromatische Aldehyde beschränkt. Darüber hinaus ist die schwierigere Zugänglichkeit dieses Enzyms ein gravierender Nachteil für technische Anwendungen. In zukünftigen Entwicklungen bei der Synthese optisch aktiver Cyanhydrine sollte deshalb vorrangig das Spektrum von Substraten von $(S)$-Oxynitrilasen erweitert und die Zugänglichkeit der Enzyme verbessert werden. Eine Erweiterung des Substratspektrums könnte durch das Auffinden und die Erschließung weiterer $(S)$-Oxynitrilasen aus anderen Quellen (Pflanzen oder Insekten) erfolgen. Für die technische Nutzung dieser Enzyme erscheint ihre Klonierung und Überexpression in effizienteren Produktionsorganismen zur Erreichung beider Ziele aussichtsreicher.

Gezielte Veränderungen der aktiven Zentren von Oxynitrilasen, die für eine Optimierung der Substratselektivitäten notwendig sind, können nicht ohne Kenntnis der Enzymstrukturen vorgenommen werden. Die Bestimmung der Kristallstrukturen von Oxynitrilasen, über die bisher nichts bekannt ist, wird deshalb eine weitere wichtige Aufgabe für zukünftige Anwendungen von Oxynitrilasen in der Synthese sein.

Dem Engagement der in diesem Artikel zitierten Mitarbeiterinnen und Mitarbeiter, die mit Begeisterung die Brücke zwischen organischer Synthese und Enzymologie geschlagen haben, ist unser Beitrag zur erfolgreichen Erschließung chiraler Cyanhydrine fü Anwendungen in der Synthese zu verdanken. Mein besonderer Dank gilt Dr. Thomas Ziegler und Dr. Siegfried Förster, die seit Beginn an der Entwicklung der in diesem Aufsatz zusammengefaßten Arbeiten großen Anteil hatten. Frau Dr. Angelika Baro war maßgehlich an der Abfassung dieses Artikels beteiligt, wofür ich ihr herzlich danke. Dem Bundesministerium für Forschung und Technologie (BMFT) gebührt Dank für die großzügige finanzielle Förderung im Rahmen des ,Zentralen Schwerpunktprogrammes Bioverfahrenstechnik Stuttgart". Dem Fonds der Chemischen Industrie danke ich für die stete Förderung.

Eingegangen am 25. Oktober 1993 [A 33]

[1] a) F. Effenberger, NATO ASI Ser. Ser. C 1992,381, 25-33 [Chem. Abstr. 1993 $118,254088 \mathrm{v}]$; b) F. Effenberger in Enzyme Catalysis in Organic Synthesis (Hrsg. : K. Drauz, H. Wakmann), VCH, Weinheim, im Druck.

[2] a) C. G. Kruse, J. Brussee, A. van der Gen, Spec. Chem. 1992, 12, 184-192 [Chem. Abstr. 1992, 117, 150273q]; b) C. G. Kruse, H. W. Geluk. G. J. M. van Scharrenburg, Chim. Oggi 1992, 10, 59-63 [Chem. Abstr. 1992, 117, 113935m]: c) C. G. Kruse in Chirality in Industry (Hrsg. A. N. Collins, G. N. Sheldrake, J. Crosby). Wiley, New York, 1992, S. 279299.

[3] W. R. Jackson, H. A. Jacobs, G. S. Jayatilake, B. R. Matthews, K. G. Watson, Aust. J. Chem. 1990, 43, 2045-2062.

[4] a) J. D. Elliott, V. M. F. Choi, W. S. Johnson, J. Org. Chem. 1983, 48, 2294 2295 ; b) M. T. Reetz, M. W. Drewes, K. Harms, W. Reif, Tetrahedron Leit. 1988, 29, 3295-3298; c) M. T. Reetz, M. W. Drewes, A. Schmitz, X. Holdgruen, T. Wuensch, J. Binder, Philos. Trans. R. Soc, London A 1988, 326, $573-578$ [Chem. Abstr. 1989, 111, 77550x].

[5] a) R. Herranz, J. Castro-Pichel, T. Garcia-Lopez, Synthesis 1989, 703-706; b) J. L. Garcia Ruano, A. M. Martin Castro, J. H. Rodriguez, Tetrahedron Lett. 1991, 32, 3195-3198.
[6] a) M. Hayashi, T. Matsuda, N. Oguni, J. Chem. Soc. Chem. Commun. 1990, 1364-1365; b) M. Hayashi, T. Matsuda, N. Oguni, J. Chem. Soc. Perkin Trans. $1,1992,3135-3140 ;$ c) M. Hayashi, Y. Miyamoto, T. Inoue, N. Oguni, J. Org. Chem. 1993, 58, 1515 1522; d) K. Narasaka, T. Yamada, H. Minamikawa, Chem. Lett. 1987, 2073-2076; e) H. Minamikawa, S. Hayakawa, T. Yamada, N. Iwasawa, K. Narasaka, Bull. Chem. Soc. Jpn. 1988, 61, 4379-4383.

[7] a) H.-J. Altenbach in Orgunic Synthesis Highlights (Hrsg.: J. Mulzer, H.-J. Altenbach, M. Braun, K. Krohn, H.-U. Reissig), 1991, S. 66-70; b) D. M. Dalton, C. M. Garner, J. M. Fernández, J. A. Gladysz, J. Org. Chem. 1991, 56, 68236829 ; c) S. Kobayashi, Y. Tsuchiya, T. Mukaiyama, Chem. Lett. 1991, 541-544; d) E. J. Corey, Z. Wang, Tetrahedron Lett. 1993, 34, 4001-4004.

[8] a) J. Oku. N. Ito, S. Inoue, Makromol. Chem. 1979, 180,1089-1091; b) J. Oku, S. Inoue, J. Chem. Soc. Chem. Commun. 1981, 229 230; c) M. North, Synlett 1993, $807-820$.

[9] L. Rosenthaler, Biochem. Z. 1908, 14, 238-253.

[10] a) V. K. Krieble, W. A. Wieland, J. Am. Chem. Soc. 1921, 43, 164-175; b) H. Albers, K. Hamann, Biochem. Z. 1932, 255, 44-65; c) H. Albers, K. Hamann, ibid. 1934, 269, 14-25.

[11] a) W. Becker, U. Benthin, E. Eschenhof, E. Pfeil, Biochem. Z. 1963, 337, 156166; b) W. Becker, E. Pfeil, ibid. 1966, 346, 301-321.

[12] a) W. Becker, H. Freund, E. Pfeil, Angew. Chem. 1965, 77, 1139; Angew. Chem. Int. Ed. Engl. 1965, 4, 1079; b) W. Becker, E. Ifeil, J. Am. Chem. Soc. 1966, 88 , 4299-4300; c) E. P[eil, W. Becker, DE-B 1300111 , 1969 [Chem. Abstr. 1970, 72, P3061t].

[13] a) F. Effenberger, T. Ziegler, S. Förster, Angew. Chem. 1987, 99, 491-492; Angew. Chem. Int. Ed. Engl. 1987, 26, 458-460; b) F. Effenberger, T. Ziegler, S. Förster (Degussa AG), DE-B 3701383,1988 [Chem. Abstr. 1989, 110, $74845 \mathrm{r}$; c) T. Ziegler, B. Hörsch, F. Effenberger, Synthesis 1990, 575-578.

[14] a) C. Laque, J. Tramper, M. D. Lilly, Biocatalysis in Organic Media, Studies in Organic Chemistry, Bd. 29, Elsevier, Ansterdam, 1987; b) S. Fukui, A. Tanaka, Endeavour 1985,9, 1017 [Chem. Abstr. 1985, 103,33788m]; c) A. M. Klibanov, Acu. Chem. Res. 1990, 23,114-120; d) K. R. Natarajan, J. Chem. Educ. 1991, 68, 13 16; e) K. Faber, Biotransformations in Organic Synthesis, Springer, Berlin, 1992, S. $248-282$.

[15] a) F. Effenberger, B. Spachmann, S. Förster, B. Bauer, J. Wonner, unveröffentlichte Ergebnisse; b) B. Bauer, H. Strathmann. F. Effenberger, DE-B 4041896. 1991 [Chem. Abstr. 1992,116, 150138d].

[16] E. Wehtje, P. Adlercreutz, B. Mattiasson, Biotechnol. Bioeng. 1990, 36, 39-46.

[17] a) U. Kragl, U. Niedermeyer, M.-R. Kula, C. Wandrey, Ann. N. Y. Acad. Sci. 1990, 613,167-175 [Chem. Abstr. 1991, 115, 181477x]; b) U. Niedermeyer, U. Kragl, M.-R. Kula, C. Wandrey, K. Makryaleas, K. Drau (Degussa AG), Eur. Pat. Appl. EP 0326063 A2, 1989 [Chem. Abstr. 1990, 112, 234012p].

[18] a) J. Brussee, E. C. Roos, A. van der Gen, Tetrahedron Leit. 1988, 29, 4485 4488 ; b) J. Brussee, W. T. Loos, C. G. Kruse, A. van der Gen, Tetrahedron 1990 , 46, 979-986; c) P. Zandbergen, J. van der Linden, J. Brussee, A. van der Gen, Synth. Commun. 1991, 21, 1387-1391.

[19] a) V. I. Ognyanov, V. K. Datcheva, K. S. Kyler, I. Am. Chem. Soc. 1991, 113, $6992-6996$; b) T. T. Huuhtanen, L. T. Kanerva, Tetrahedran Asymmetry 1992, $3,1223-1226$

[20] F. Effenberger, J. Eichhorn, unveröffentlichtc Ergebnisse.

[21] a) C. Bove, E. E. Conn, J. Biol. Chem. 1961, 236, 207-210; b) M. K. Seely, R. S. Criddle, E. E. Conn, ibid. 1966, 241, 4457-4462.

[22] a) I. Jansen, R. Woker, M.-R. Kula, Biotechnol. Appl. Biochem. 1992, 15, 9099; b) H. Wajant, K.-W. Mundry, Plant Sci. Limerick Irel 1993, 89, 127-133; c) E. Smitskamp-Wilms, J. Brussee, A. van der Gen, G. J. M. van Scharrenburg, J. B. Sloothaak, Recl. Trav. Chim. Pays-Bas 1991, 110, 209-215.

[23] F. Effenberger, B. Hörsch, S. Förster, T. Ziegler, Tetrahedron Lett. 1990, 31, $1249-1252$.

[24] U. Niedermeyer, M.-R. Kula, Angew. Chem. 1990, 102, 423 424; Angew. Chem. Int. Ed. Engl. 1990, 29, 386-387.

[25] N. Klempier, H. Griengl. M. Hayn, Tetrahedron Lett. 1993, 34, 4769-4772.

[26] a) S. Förster, F. Effenberger, unveröffentlichte Ergebnisse; b) H. Wajant, H. Böttinger, K.-W. Mundry, Biotechnol. Appl. Biochem. 1993, 18, 75-82.

[27] D. A. Livingston, J. E. Petre, C. L. Bergh, J. Am. Chem. Soc. 1990, 112, 64496450 .

[28] N. S. Batra, E. Brunet, Tetrahedron Lett. 1993, 34, 711-714.

[29] J. L. Garcia Ruano, A. M. Martin Castro, J. II. Rodriguez, J. Org. Chem. 1992, $57,7235-7241$.

[30] M. T. Reetr, K. Kesseler, A. Jung, Angew. Chem. 1985, 97, 989-990; Angew. Chem. Inl. Ed. Engl. 1985, 24, $989-990$.

[31] H. Ohta, Y. Kimura, Y. Sugano, T. Sugai, Tetrahedron 1989, 45, 5469-5476.

[32] A. Nahrstedt, Biol. Unserer Zeit 1988, 18, 105-109.

[33] a) F. Effenberger, B. Hörsch, F. Weingart, T. Ziegler, S. Kühner, Tetrahedron Letr. 1991, 32, 2605-2608; b) S. Heid, Diplomarbeit, Universität Stuttgart, 1991.

[34] a) J. Oku, N. Ito, S. Inoue, Makromol. Chem. 1982, 183, 579-586; b) A. Mori, Y. Ikeda, K. Kinoshita, S. Inoue, Chem. Lett. 1989,2119-2122; c) K. Tanaka, A. Mori, S. Inoue, J. Org. Chem. 1990, 55, 181-185.

[35] a) S. Asada, Y. Kobayashi, S. Inoue, Makromol. Chem. 1985, 186, 1755-1762; b) Y. Kobayashi, S. Asada, I. Watanabe, H. Hayashi, Y. Motoo, S. Inoue, Bull. Chem. Soc. Jpn. 1986, 59, 893-895. 
[36] a) W. R. Jackson, G. S. Jayatilake, B. R. Matthews, C. Wilshire, Aust. J. Chem 1988, 41, 203-213; b) B. R. Matthews, W. R. Jackson, G. S. Jayatilake. C. Wilshire, H. A. Jacobs, ibid. 1988, 41, 1697-1709; c) W. Dong, W. L. Petty (Shell Oil Co.), US-A 535500, 1983 [Chem. Ab́str. 1985, 103, 196420z].

[37] a) H. Danda, H. Nishikawa, K. Otaka, J. Org. Chem. 1991, 56, 6740-6741; b) H. Danda, Synlett 1991, 263-264; c) S. D. Rychnovsky, Chemtracts: Org. Chem. 1992, 5, 102-105 [Chem. Abstr. 1992, 117, 150608c].

[38] K. Naumann, Chemistry of Plant Protection, 5: Synthetic Pyrethroid Insecticides: Chemistry and Patents, Springer, Berlin, 1990, S. 110-141.

[39] a) M. North, Tetrahedron 1992, 48,5509-5522; b) D. Callant, B. Coussens, T. v. d. Maten, J. G. de Vries, N. Koen de Vries, Tetrahedron Asymmetry 1992, 3, 401-414; c) H. J. Kim, W. R. Jackson, ibid. 1992, 3, 1421-1430; d) D. J. P. Hogg, M. North, Tetrahedron 1993, 49, 10791090.

[40] a) A. Mori, H. Ohno, H. Nitta, K. Tanaka, S. Inoue, Synlett 1991, 563-564; b) H. Ohno, H. Nitta, K. Tanaka, A. Mori, S. Inoue, J. Org. Chem. 1992, 57, $6778-6783$.

[41] F. Effenberger, B. Gutterer, T. Ziegler, E. Eckhardt, R. Aichholz, Liebigs Ann. Chem. 1991, 47-54, zit. Lit.

[42] a) N. Matsuo, N. Ohno, Tetrahedron Lett. 1985, 26, 5533-5534; b) A. van Almsick, J. Buddrus, P. Hönicke-Schmidt, K. Laumen, M. P. Schneider, $J$. Chem. Soc. Chem. Commun. 1989, 1391-1393.

[43] a) H. Ohta, Y. Miyamac, G. Tsuchihashi, Agric. Biol. Chem. 1986, 50, 3181 3184; ibid. 1989, 53, 215-222; b) H. Hirohara, S. Mitsuda, E. Ando, R. Komaki, Stud. Org. Chem. (Amsterdam) 1985, 22,119-134.

[44] a) Y. F. Wang, S. T. Chen, K. K. C. Liu, C. H. Wong, Tetrahedran Lett. 1989 $30,1917-1920$; b) S. H. Hsu, S. S. Wu, Y. F. Wang, C. H. Wong, ibid. 1990, 31 , $6403-6406$.

[45] a) M. Inagaki, J. Hiratake, T. Nishioka, I. Oda, J. Am. Chem. Soc. 1991. 113, 9360-9361 ; b) M. Inagaki, A. Hatanaka, M. Mimura, J. Hiratake, T. Nishioka, J. Oda, Bull. Chem. Soc. Jpn. 1992, 65, 111-120; c) M. Inagaki, J. Hiratake. T. Nishioka, J. Oda, J. Org. Chem. 1992, 57, 5643-5649.

[46] H. S. Bevinakatti, A. A. Banerji, R. V. Newadkar, J. Org. Chem. 1989, 54, $2453-2455$.

[47] a) D. A. Evans, M. M. Morrissey, R. L. Dorow, J. Am. Chem. Soc. 1985, 107 4346-4348; b) H. C. Brown, J. Chandrasekharan, P. V. Ramachandran, $J$. Org. Chem. 1986, 5t, 3394-3396; c) E. J. Corey, J. O. Link, Tetrahedron Lett. 1992, 33, 3431-3434; d) K. Mikami, M. Terada, T. Nakai, J. Am. Chem. Soc. 1990. $112,3949-3954$.

[48] a) S. Servi, Synihesis 1990, 1-25; b) S. Tsuboi, E. Nishiyama, H. Furutani, M Utaka, A. Takeda, J. Org. Chem. 1987, 52, 1359-1362.

[49] a) M.-J. Kim, G. M. Whitesides, J. Am. Chem. Soc. 1988, 110, 2959-2964; b) M.-R. Kula, C. Wandrey, Methods Enzymol. 1987, 136, 9-21 ; c) M.-J. Kim, J. Y. Kim, J. Chem. Soc. Chem. Commur. 1991, 326-327.

[50] a) P. Kalaritis, R. W. Regenye, J. J. Partridge, D. L. Coffen, J. Org. Chem. 1990, 55, 812-815; b) T. Sugai, H. Ohta, Tetrahedron Lett. 1991, 32, 7063-7064

[51] a) U. Burkard, F. Effenberger, Chem. Ber. 1986, 119, 1594-1612; b) H. Kunz, H.-G. Lerchen, Tetrahedron Lett. 1987, 28, 1873-1876.
[52] a) S. K. Massad, L. D. Hawkins, D. C. Baker, J. Org. Chem. 1983, 48, 51805182 ; b) J.-R. Schwark, D. Hoppe, Synthesis 1990, 291-294; c) Y. Kobayashi, M. Takase, Y. Ito, S. Terashima, Bull. Chem. Soc. Jpn. 1989, 62, 3038-3040; d) M. Larcheveque, Y. Petit, Bull. Soc. Chim. Fr. 1989.130-139; e) E. J. Corey, G. B. Jones. Tetrahedron Lett. 1991, 32, 5713-5716.

[53] a) W. Althoff, R. Karsdorf, P. Tinapp, Arch. Pharm. (Weinheim Ger.) 1981, $314,518-524$; b) B. R. Matthews, H. Gountzos, W. R. Jackson, K. G. Watson, Tetrahedron Lett. 1989, 30, 5157-5158.

[54] F. Effenberger, M. Hopf, T. Ziegler, J. Hudelmayer, Chem. Ber. 1991, 124, 16511659

[55] a) J. Engel, Chem.-Ztg. 1982, 106,169-183; b) A. Kleemann, J. Engel, Pharmazeutische Wirkstoffe, Synthesen, Patente, Anwendungen, 2. Aufl., Thieme, Stuttgart, 1982, sowie Ergänzungsband 1987; c) E. J. Corey, J. O. Link, Tetrahedron Lett. 1990, 31, 601-604; d) J. Org. Chem. 1991, 56, 442-444; e) M. T. Reetz, M. W. Drewes, A. Schmitz. Angew. Chem. 1987, 99, 1186-1188; Angew. Chem. Int. Ed. Engl. 1987, 26, 1141-1143; f) M. T. Reetz, M. W. Drewes, K. Lennick. A. Schmitz, X. Holdgrün, Tetrahedron Asymmetry 1990, 1 , $375-378 ;$ g) T. Ishizuka, S. Ishibuchi, T. Kunieda, Tetrahedron 1993, 49, 1841 1852 .

[56] N. Matsuo, N. Ohno. Tetrahedron Lett. 1985, 26, 5533 - 5534.

[57] L. R. Krepski, K. M. Jensen, S. M. Heilmann, J. K. Rasmussen, Synthesis 1986, $301-303$.

[58] a) J. Brussee, F. Dofferhoff, C. G. Kruse, A. van der Gen, Tetrahedron 1990, 46, 1653-1658; b) W. R. Jackson, H. A. Jacobs, B. R. Matthews, G. S. Jayatilake, K. G. Watson. Tetrahedron Lett. 1990, 31, 1447-1450.

[59] F. Effenberger, B. Gutterer, T. Ziegler, Liebigs Ann. Chem. 1991, 269-273.

[60] G. Cainelli, E. Mezzina, M. Panunzio, Tetrahedron Lett. 1990, 31, $3481-$ 3484.

[61] a) P. Zandbergen, A. M. C. H. van den Nieuwendijk, J. Brussee, A. van der Gen. Tetrahedron 1992, 48, 3977-3982; b) J. Brussee, A. van der Gen, Recl. Trav. Chim. Pays-Bas 1991, 110, 25-26.

[62] P. Zandbergen, J. Brussee, A. van der Gen, C. G. Kruse, Tetrahedron Asymmetry 1992, 3, $769-774$.

[63] J. Brussee, R. A. T. M. van Benthem, C. G. Kruse, A. van der Gen, Tetrahedron Asymmetry 1990, $1,163-166$.

[64] a) F. Effenberger, U. Stelzer, Angew. Chem. 1991, 103, 866-867; Angew. Chem. Int. Ed. Engl. 1991, 30,873-874; b) F. Effenberger, U. Stelzer, Chem. Ber. 1993, $126,779-786$.

[65] a) F. Effenberger, U. Burkard, J. Willfahrt, Liehigs Ann. Chem. 1986, 314-333; b) U. Azzena, G. Delogu, G. Melloni, O. Piccolo, Tetrahedron Lett. 1989, 30, $4555-4558$; c) P. R. Fleming, K. B. Sharpless, J. Org. Chem. 1991, 56, $2869-$ 2875 .

[66] a) I. A. Smith, Ber. Dtsch. Chem. Ges. B 1938, 71, 634-643; b) K. Ichimura, M. Ohta, Bull. Chem. Soc. Jpn. 1970, 43, 1443-1450.

[67] U. Stelzer, F. Effenberger, Tetrahedron Asymmetry 1993, 4, 161-164.

[68] E. G. J. C. Warmerdam, J. Brussee, C. G. Krusc, A. van der Gen, Tetrahedron $1993,49,1063-1070$. 